\title{
CONTINUOUS-TIME RANDOM WALK BETWEEN LÉVY-SPACED TARGETS IN THE REAL LINE
}

\author{
ALESSANDRA BIANCHI, MARCO LENCI, AND FRANÇOISE PÈNE
}

\begin{abstract}
We consider a continuous-time random walk which is defined as an interpolation of a random walk on a point process on the real line. The distances between neighboring points of the point process are i.i.d. random variables in the normal domain of attraction of an $\alpha$-stable distribution with $0<\alpha<1$. This is therefore an example of a random walk in a Lévy random medium. Specifically, it is a generalization of a process known in the physical literature as Lévy-Lorentz gas. We prove that the annealed version of the process is superdiffusive with scaling exponent $1 /(\alpha+1)$ and identify the limiting process, which is not càdlàg. The proofs are based on the technique of Kesten and Spitzer for random walks in random scenery.

Mathematics Subject Classification (2010): 60G50, 60F05 (82C41, 60G55, 60F17).

Keywords: Lévy-Lorentz gas, random walk on point process, anomalous diffusion, Lévy random medium, stable processes, random walk in random scenery.
\end{abstract}

\section{INTRODUCTION}

In this paper we study a continuous-time random walk in a random medium on the real line. The random medium is given by a marked point process in which the distances between consecutive points are i.i.d. variables taken in the basin of attraction of an $\alpha$-stable distribution, with $0<\alpha<1$. The marked points are referred to as targets. A particle travels in $\mathbb{R}$ with unit speed visiting the sequence of targets selected by an independent random walk $\left(S_{n}, n \in \mathbb{N}\right)$. This random walk is called the underlying random walk.

So, for example, if the realization of $\left(S_{n}\right)$ is $(0,2,-3,-1, \ldots)$, the particle will travel with velocity 1 from the origin $O$ to the second target to the right of $O$. Then it will instantaneously change its velocity to -1 to travel toward the third target to the left of $O$. Then it will travel with velocity 1 toward the first target to the left of $O$, and so on.

Since the distances between targets are fat-tailed variables (their first moment is infinite), the trajectories of the process occasionally experience extremely long inertial segments, leading one to believe that the process is superdiffusive. By that we mean that it scales like a power of time with exponent bigger than $1 / 2$. The purpose of the paper is to show this in a very specific sense.

More precisely, if $X(t) \equiv X^{\omega}(t)$ denotes our process, with the label $\omega$ representing the random medium, we prove that the finite-dimensional distributions of $\left(n^{-1 /(\alpha+1)} X(n t), t \geq 0\right)$, w.r.t. both the random medium and the random dynamics, 
converges to those of a certain process which we identify (Theorem 2.1). In other words, we prove an annealed generalized CLT for the finite-dimensional distributions of $(X(t), t \geq 0)$.

Processes of this kind have wide application in the physical sciences, where they are used as models for anomalous diffusion; see [SZU, [KRS, ZDK, CGLS, VBB] and references therein. Indeed, in many real-world applications (in statistical physics, optics, epidemics, etc.), the long inertial segments, or ballistic flights, that cause superdiffusion are not due to an anomalous mechanism that governs the dynamics of the agent (a particle, a photon, an animal, etc.) but rather to the complexity of the medium in which the motion occurs. An example is molecular diffusion in porous media [Le] (see the reference lists of [Le, [BFK, [BCV] for more examples). As a matter of fact, the model presented here is a generalization of the so-called Lévy-Lorentz gas, which was introduced in [BFK] precisely as a one-dimensional toy model for transport in porous media. (The Lévy-Lorentz gas is the case where the underlying random walk is a simple symmetric random walk.)

In the language of probability it makes sense to call the process at hand a continuous-time random walk in a Lévy random medium, meaning that it is the medium that causes the long ballistic flights. We occasionally also use the expression 'random walk in a Lévy random environment' to lay emphasis on the analogies between our process and the random walks in random environment - even though the two types of processes are technically different.

A rigorous study of our system was initiated in [BCLL] where the authors considered the easier case where the distances between the targets have finite mean and infinite variance. This includes all random variables with distribution in the basin of attraction of an $\alpha$-stable law with $1<\alpha<2$. For this regime, subject to certain hypotheses, they prove a quenched normal CLT, that is, for a.e. realization $\omega$ of the medium, $t^{-1 / 2} X^{\omega}(t)$ converges, as $t \rightarrow \infty$, to a Gaussian variable independent of $\omega$. This implies in particular the annealed normal CLT, confirming and adding on some of the predictions of [BCV] for the Lévy-Lorentz gas.

Our main result in this paper confirms and extends some other predictions of [BCV] and for the first time, to our knowledge, prove superdiffusion for some processes in Lévy random media. The scaling we obtain is also in agreement with the one that has been identified recently for a related model ACOR. Our proofs are based on an adaptation of the technique of Kesten and Spitzer for random walks in random scenery [KS]. This explains why our limit processes involve the so-called Kesten-Spitzer process $\Delta$.

The paper is organized as follows: In Section 2 we give the precise definitions of all the processes associated with our random walk, and present our most important results (Theorems 2.1 and 2.2). In Section 3 we prove these results by means of what we call our Main Lemma (Lemma 3.1). The Main Lemma is then proved in Section 4, using ideas from the theory of random walks in random scenery.

Acknowledgments. F. Pène is supported by the IUF and by the ANR Project MALIN (ANR-06-TCOM-0003). M. Lenci's research is part of his activity within the Gruppo Nazionale di Fisica Matematica (INdAM). We also wish to thank the 
American Institute of Mathematics for the Workshop "Stochastic Methods for NonEquilibrium Dynamical Systems", where this work was initiated.

\section{Setup}

Let $\zeta:=\left(\zeta_{j}, j \in \mathbb{Z}\right)$ be a sequence of i.i.d. positive random variables, the common distribution of which belongs to the normal basin of attraction of an $\alpha$-stable distribution, with $0<\alpha<1$. This means that, as $n \rightarrow \infty, n^{-1 / \alpha} \sum_{j=1}^{n} \zeta_{j}$ converges in distribution to a variable $Z_{1}$, which must then be positive and non-integrable. The recursive sequence of definitions

$$
\omega_{0}:=0, \quad \omega_{k}-\omega_{k-1}:=\zeta_{k}, \quad \text { for } k \in \mathbb{Z} .
$$

determines a marked point process $\omega:=\left(\omega_{k}, k \in \mathbb{Z}\right)$ on $\mathbb{R}$, which we call the random medium or random environment, or simply the environment. Each point $\omega_{k}$ will be called a target. The set of all possible environments is denoted $\Omega_{\mathrm{en}}$, and the law on it $P$.

Let $S:=\left(S_{n}, n \in \mathbb{N}\right)$ be a $\mathbb{Z}$-valued random walk starting from $S_{0}:=0$ with centered i.i.d. increments $\xi_{j}:=S_{j}-S_{j-1}$, for $j \in \mathbb{Z}^{+}$. We assume that there exists $\gamma>2 / \alpha$ such that the absolute moment of $\xi_{j}$ of order $\gamma$ is finite (this assumption is used in Lemma 4.3). This implies that the variance of $\xi_{j}$, which we denote $v_{\xi}$, is also finite. In order for the problem to make sense, we also assume $v_{\xi}>0$. We refer to $S$ as the underlying random walk and call $Q$ its distribution (on $\mathbb{Z}^{\mathbb{N}}$ endowed with the $\sigma$-algebra generated by cylinders).

Given $\omega \in \Omega_{\mathrm{en}}$, we define the so-called random walk on the point process to be, for all $n \in \mathbb{N}$,

$$
Y_{n} \equiv Y_{n}^{\omega}:=\omega_{S_{n}}
$$

In simple terms, $Y:=\left(Y_{n}, n \in \mathbb{N}\right)$ performs the same jumps as $S$, but on the points of $\omega$.

The process we are interested in here is the continuous-time random walk $X \equiv$ $X^{\omega}:=\left(X^{\omega}(t), t \geq 0\right)$ whose trajectories interpolate those of the walk $Y$ and have unit speed (save at collision times). Formally it can be defined as follows: Given a realization $\omega$ of the medium and a realization $S$ of the dynamics, we define the sequence of collision times $T_{n} \equiv T_{n}(\omega, S)$ via

$$
T_{0}:=0, \quad T_{n}:=\sum_{k=1}^{n}\left|\omega_{S_{k}}-\omega_{S_{k-1}}\right|, \quad \text { for } n \geq 1 .
$$

Since the length of the $n^{\text {th }}$ jump of the walk is given by $\left|\omega_{S_{n}}-\omega_{S_{n-1}}\right|, T_{n}$ represents the global length of the trajectory up to the $n^{\text {th }}$ collision.

Finally, $X(t) \equiv X^{\omega}(t)$ is defined by the equations

$$
X(t):=Y_{n}+\operatorname{sgn}\left(\xi_{n+1}\right)\left(t-T_{n}\right), \quad \text { for } t \in\left[T_{n}, T_{n+1}\right) .
$$

Notice that $\xi_{n+1}=0 \Leftrightarrow S_{n+1}=S_{n} \Leftrightarrow T_{n+1}=T_{n}$. Therefore the definition (2.4) is never used in the case $\xi_{n+1}=0$, making the value of $\operatorname{sgn}(0)$ irrelevant there. More importantly, the self-jumps of the underlying RW, i.e., $S_{n+1}=S_{n}$, are not seen by the continuous-time process $X$. This implies that one can remove any lazy 
component of $S$ by replacing the common distribution of the $\xi_{j}$ by the conditional distribution of $\xi_{1}$ given $\xi_{1} \neq 0$ (this is well defined since $v_{\xi}>0$ ). We thus assume from now on that $Q\left(\xi_{j}=0\right)=0$.

Also without loss of generality we assume that $\xi_{1}$, equivalently $S$, is not supported on a proper subgroup if $\mathbb{Z}$. If it were supported, say, on $d \mathbb{Z}$, with $d \in \mathbb{N}, d \geq 2$, one could divide $S$ by $d$ and replace $\zeta_{j}$ with $\sum_{k=(j-1) d+1}^{j d} \zeta_{k}$. The resulting continuoustime walk would be a simple rescaling of the original process $X$.

The law that embraces all the random aspects of the process is the product probability $\mathbb{P}:=P \times Q$ on $\Omega_{\text {en }} \times \mathbb{Z}^{\mathbb{N}}$. The probability $\mathbb{P}$ is sometimes called the annealed or averaged law.

Our main result is the finite-dimensional generalized CLT for the family of processes

$$
\left(\bar{X}^{(q)}(s), s \geq 0\right):=\left(\frac{X(s q)}{q^{1 /(\alpha+1)}}, s \geq 0\right),
$$

where $q \in \mathbb{R}^{+}$plays the role of the scaling parameter. In order to state it, we need some preliminary notation. Let $(B(t), t \geq 0)$ be a Brownian motion such that $B(t)$ has mean 0 and variance $v_{\xi} t$, and denote by $\left(L_{t}(x), x \in \mathbb{R}\right)$ its corresponding local time. Let $\left(Z_{ \pm}(x), x \geq 0\right)$ be two i.i.d. càdlàg $\alpha$-stable processes with independent and stationary increments such that $Z_{ \pm}(0) \equiv 0$ and $Z_{ \pm}(1)$ is distributed like $Z_{1}$, cf. beginning of the section. Notice that the processes $Z_{ \pm}$are strictly increasing subordinators.

Assume that the processes $B, Z_{+}$and $Z_{-}$are mutually independent and consider the process $\Delta:=(\Delta(t), t \geq 0)$ given by

$$
\Delta(t):=\mu_{\xi}\left(\int_{0}^{+\infty} L_{t}(x) d Z_{+}(x)+\int_{0}^{+\infty} L_{t}(-x) d Z_{-}(x)\right),
$$

with $\mu_{\xi}:=\mathbb{E}\left(\left|\xi_{1}\right|\right)$. The above is well-defined because $x \mapsto L_{t}(x)$ is almost surely continuous and compactly supported. Also, let us define $(Z(x), x \in \mathbb{R})$ to be

$$
Z(x):=\left\{\begin{array}{cc}
Z_{+}(x), & x \geq 0 \\
-Z_{-}(-x), & x<0
\end{array}\right.
$$

Observe moreover that $\Delta$ is a.s. continuous and strictly increasing.

Theorem 2.1. Under the law $\mathbb{P}$, the finite dimensional distributions of $\left(\bar{X}^{(q)}(s), s \geq\right.$ $0)$ converge, as $q \rightarrow \infty$, to those of $\left(Z \circ B \circ \Delta^{-1}(s), s \geq 0\right)$.

The process $\Delta$, often called the Kesten-Spitzer process, was introduced in [KS] as the limit, for $n \rightarrow \infty$, of the processes

$$
\left(\frac{1}{n^{(1+\alpha) / 2 \alpha}} \sum_{k=1}^{\lfloor n t\rfloor} \zeta_{S_{k}}, t \in \mathbb{R}^{+}\right)
$$

where $\lfloor a\rfloor$ denotes the largest integer less than or equal to $a \in \mathbb{R}$. The sum $\sum_{k} \zeta_{S_{k}}$ is referred to as a random walk in the random scenery $\zeta$; see also [Bo1, Bo2]. In the present context $\Delta$ appears as the limit of the suitably rescaled collision time processes, as clarified by the next result, which also gives the asymptotic behavior of $Y$, 
the random walk on the point process. (The recent reference [MS] also investigates limit theorems for $Y$ in the case $\alpha \in(1,2)$, and with some restrictions. This process is of course simpler than the actual Lévy-Lorentz gas.)

Theorem 2.2. Under $\mathbb{P}$, the (joint) finite dimensional distributions of

$$
\left(\left(\frac{T_{\lfloor t q\rfloor}}{q^{(1+\alpha) / 2 \alpha}}, \frac{Y_{\lfloor t q\rfloor}}{q^{1 / 2 \alpha}}\right), t \geq 0\right)
$$

converge, for $q \rightarrow \infty$, to the corresponding distributions of $((\Delta, Z \circ B), t \geq 0)$.

As a last remark for this section, observe that neither Theorem 2.1 nor Theorem 2.2 can be extended to a functional limit theorem w.r.t. any Skorokhod topology, since the limit processes do not belong to the Skorokhod space. Indeed $Z \circ B \circ \Delta^{-1}$ and $Z \circ B$ have discontinuities without one-sided limits. This can be seen as follows: If $x_{0}$ is a jump discontinuity of $Z$, which exists almost surely, and $B\left(t_{0}\right)=x_{0}$, then almost surely $B(t)$ oscillates around $x_{0}$, both as $t \rightarrow t_{0}^{+}$and as $t \rightarrow t_{0}^{-}$. Therefore neither limit

$$
\lim _{t \rightarrow t_{0}^{ \pm}} Z \circ B(t)
$$

exists. The situation is analogous for $Z \circ B \circ \Delta^{-1}$.

Heuristically, the cause of this phenomenon is that the gaps between targets are non-integrable i.i.d. variables. Therefore, from time to time, the walker will encounter a gap of the same order as the sum of all the gaps visited till then. The variation in position achieved upon traveling that gap will thus be of finite order in the limit. Since, as $q \rightarrow \infty$, time shrinks by a factor $q^{-1}$ and space by a factor

$q^{-1 /(\alpha+1)} \ll q^{-1}$, the trajectory segments corresponding to these exceptionally long gaps become jump discontinuities. On the other hand, some of these gaps will be traveled on, back and forth, a larger and larger number of times, creating in the limit an accumulation of jump discontinuities of the same size.

\section{Rescaled PROCESSES}

At the core of the proofs lies a functional CLT for the joint distribution of all the processes involved here: the underlying random walk, the point process and the collision time process. In this Section we state this limit theorem, later referred to as the Main Lemma, and use it to derive Theorems 2.1 and 2.2.

Fix a value $q \in \mathbb{R}^{+}$of the scaling parameter and let the processes $\left(\bar{\omega}^{(q)}(x), x \in \mathbb{R}\right)$, $\left(\bar{S}^{(q)}(t), t \geq 0\right),\left(\bar{T}^{(q)}(t), t \geq 0\right)$ be defined by

$$
\begin{aligned}
\bar{\omega}^{(q)}(x) & :=\frac{\omega_{\lfloor x \sqrt{q}\rfloor}}{q^{1 / 2 \alpha}} ; \\
\bar{S}^{(q)}(t) & :=\frac{S_{\lfloor t q\rfloor}}{\sqrt{q}} ; \\
\bar{T}^{(q)}(t) & :=\frac{T_{\lfloor t q\rfloor}}{q^{(\alpha+1) / 2 \alpha}} .
\end{aligned}
$$


Convention. Throughout the paper, the notation $[a, b]$ denotes the closed interval between the real numbers $a$ and $b$, irrespective of their order.

This is our Main Lemma:

Lemma 3.1. Under $\mathbb{P}$, as $q \rightarrow \infty$, the joint process

$$
\left(\left(\bar{\omega}^{(q)}(x), x \in \mathbb{R}\right),\left(\bar{S}^{(q)}(t), t \geq 0\right),\left(\bar{T}^{(q)}\left(t^{\prime}\right), t^{\prime} \geq 0\right)\right)
$$

converges to

$$
\left((Z(x), x \in \mathbb{R}),(B(t), t \geq 0),\left(\Delta\left(t^{\prime}\right), t^{\prime} \geq 0\right)\right)
$$

w.r.t. the (product) $J_{1}$-metric on $\mathbb{D}(\mathbb{R}) \times \mathbb{D}\left(\mathbb{R}_{0}^{+}\right) \times \mathbb{D}\left(\mathbb{R}_{0}^{+}\right)$. Here $\mathbb{D}(\mathbb{R})$ is the space of the trajectories $\bar{\omega}(x): \mathbb{R} \longrightarrow \mathbb{R}$ such that $(\bar{\omega}(x), x \geq 0)$ and $(-\bar{\omega}(-x), x \geq 0)$ are càdlàg; the space is endowed with the topology induced by the $J_{1}$-metric on every interval $[0, y], y \in \mathbb{R}$ (see $[\mathrm{B}$, Secs. 12 and 16]).

3.1. Proof of Theorems 2.1 and 2.2. If $f: \mathbb{R} \longrightarrow \mathbb{R}$ is a (not necessarily strictly) increasing function, let $f^{-1}(s):=\sup \{u>0: f(u)<s\}$ denote our choice for the generalized inverse of $f$.

The function

$$
t \mapsto \bar{T}^{\left(q^{2 \alpha /(\alpha+1)}\right)}(t)=\frac{T_{\left\lfloor t q^{2 \alpha /(\alpha+1)}\right\rfloor}}{q},
$$

cf. (3.3), is an increasing càdlàg function which is constant on any interval of the form $\left[q^{-2 \alpha /(\alpha+1)} n, q^{-2 \alpha /(\alpha+1)}(n+1)\right), n \in \mathbb{N}$. Therefore, given $s \in \mathbb{R}^{+}$,

$$
\left(\bar{T}^{\left(q^{2 \alpha /(\alpha+1)}\right)}\right)^{-1}(s)=q^{-2 \alpha /(\alpha+1)} \bar{n},
$$

for some $\bar{n} \equiv \bar{n}(s, q) \in \mathbb{N}$. The previous two equations imply that $T_{\bar{n}-1}<s q \leq T_{\bar{n}}$, whence, in view of (2.2) and (2.5),

$$
\bar{X}^{(q)}(s) \in\left[\frac{\omega_{S_{\bar{n}-1}}}{q^{1 /(\alpha+1)}}, \frac{\omega_{S_{\bar{n}}}}{q^{1 /(\alpha+1)}}\right] .
$$

Denoting

$$
p \equiv p(q):=q^{2 \alpha /(\alpha+1)}
$$

and using (3.5) and (3.2), we see that

$$
S_{\bar{n}}=\sqrt{p} \bar{S}^{(p)}\left(\left(\bar{T}^{(p)}\right)^{-1}(s)\right),
$$

whence, in view of (3.1),

$$
\omega_{S_{\bar{n}}}=p^{1 / 2 \alpha} \bar{\omega}^{(p)}\left(\bar{S}^{(p)}\left(\left(\bar{T}^{(p)}\right)^{-1}(s)\right)\right) .
$$

Since $p^{1 / 2 \alpha}=q^{1 /(\alpha+1)}$, 3.6 becomes

$$
\bar{X}^{(q)}(s) \in\left[\bar{\omega}^{(p)} \circ \bar{S}^{(p)}\left(\left(\bar{T}^{(p)}\right)^{-1}(s)-p^{-1}\right), \bar{\omega}^{(p)} \circ \bar{S}^{(p)} \circ\left(\bar{T}^{(p)}\right)^{-1}(s)\right] .
$$

Let $\left(q_{k}\right)_{k \in \mathbb{Z}^{+}}$be a positive sequence of real numbers such that $q_{k} \rightarrow+\infty$ as $k \rightarrow \infty$, and let $p_{k}:=p\left(q_{k}\right)$ as defined in (3.7) above. 
Assumption. From now on we assume that the convergence in the statement of Lemma 3.1, restricted to $q=p_{k}, k \rightarrow \infty$, holds almost everywhere. If this is not the case, by virtue of the Skorokhod representation theorem [D, Thm. 3], there exists a probability space where it does (for processes which have the same joint distribution as the ones used in Lemma 3.1): as we shall see, the specifics of the probability space are irrelevant. In particular, up to discarding a null set of realizations, we may assume that $B:[0,+\infty) \longrightarrow \mathbb{R}$ is continuous and $\Delta:[0,+\infty) \longrightarrow[0,+\infty)$ is continuous, strictly increasing and bijective.

Lemma 3.2. (Almost) surely, for all $s>0$,

$$
\lim _{k \rightarrow \infty}\left(\bar{T}^{\left(p_{k}\right)}\right)^{-1}(s)=\Delta^{-1}(s) .
$$

Proof. Since $\Delta$ is continuous and strictly increasing, so is the inverse $\Delta^{-1}$. For a given $s>0$ and for every sufficiently small $\theta>0$, we set $u:=\Delta^{-1}(s), x_{\theta}:=$ $\Delta^{-1}(s-\theta / 2)$ and $y_{\theta}:=\Delta^{-1}(s+\theta / 2)$, so that

$$
\Delta\left(x_{\theta}\right)=s-\frac{\theta}{2}<s=\Delta(u)<\Delta\left(y_{\theta}\right)=s+\frac{\theta}{2} .
$$

Then, for any given $\varepsilon>0$, we choose $\theta>0$ such that $u-x_{\theta}<\varepsilon / 2$ and $y_{\theta}-u<$ $\varepsilon / 2$. Moreover, we set $\varsigma:=\min \left\{\theta / 2, u-x_{\theta}, y_{\theta}-u, \varepsilon / 2\right\}$ and notice that for $k$ big enough, we have $d_{J_{1},[0, u+1]}\left(\bar{T}^{\left(p_{k}\right)}, \Delta\right)<\varsigma$, where the 1.h.s. of the inequality denotes the Skorokhod $J_{1}$-distance on the interval $[0, u+1]$. In other words, there exists a strictly increasing homeomorphism $\varphi_{k}:[0, u+1] \longrightarrow[0, u+1]$ such that, for all $v \in[0, u+1]$

$$
\begin{array}{r}
\left|v-\varphi_{k}(v)\right|<\varsigma ; \\
\left|\Delta(v)-\bar{T}^{\left(p_{k}\right)}\left(\varphi_{k}(v)\right)\right|<\varsigma .
\end{array}
$$

Hence, for $k$ big enough, one has

$$
\begin{aligned}
& \bar{T}^{\left(p_{k}\right)}\left(\varphi_{k}\left(y_{\theta}\right)\right)>\Delta\left(y_{\theta}\right)-\varsigma=s+\frac{\theta}{2}-\varsigma \geq s ; \\
& \bar{T}^{\left(p_{k}\right)}\left(\varphi_{k}\left(x_{\theta}\right)\right)<\Delta\left(x_{\theta}\right)+\varsigma=s-\frac{\theta}{2}+\varsigma \leq s,
\end{aligned}
$$

whence

$$
\begin{aligned}
& \left(\bar{T}^{\left(p_{k}\right)}\right)^{-1}(s) \geq \varphi_{k}\left(x_{\theta}\right)>x_{\theta}-\varsigma>u-\frac{\varepsilon}{2}-\varsigma \geq u-\varepsilon \\
& \left(\bar{T}^{\left(p_{k}\right)}\right)^{-1}(s) \leq \varphi_{k}\left(y_{\theta}\right)<y_{\theta}+\varsigma<u+\frac{\varepsilon}{2}+\varsigma \leq u+\varepsilon .
\end{aligned}
$$

Since $\varepsilon$ was arbitrary, this proves the stated convergence.

Observe now that $Z$ and $B$ are independent and that the discontinuities of $Z$ occur at random points whose distribution has no atom in $\mathbb{R}$. Therefore, for every $t \geq 0, Z$ is continuous at $B(t)$, equivalently, $Z$ does not have a jump at $B(t)$, with probability 1 . This ensures that the following lemma holds true for almost every realization of $(B, Z)$. 
Lemma 3.3. Let $t>0$ and consider a realization of our processes such that $Z$ is continuous at $B(t)$. For any sequence $\left(a_{k}\right) \subset \mathbb{R}^{+}$with $\lim _{k \rightarrow \infty} a_{k}=t$, we have:

$$
\lim _{k \rightarrow \infty} \bar{\omega}^{\left(p_{k}\right)}\left(\bar{S}^{\left(p_{k}\right)}\left(a_{k}\right)\right)=Z(B(t))
$$

Proof. Let $\varepsilon \in(0,1)$ and $\eta \in(0, \varepsilon)$ be such that

$$
\sup _{x:|x-B(t)|<2 \eta}|Z(B(t))-Z(x)|<\frac{\varepsilon}{2} .
$$

Also choose $\varsigma \in(0, \eta / 2)$ so that

$$
\sup _{v:|v-t|<\varsigma}|B(t)-B(v)|<\frac{\eta}{2} .
$$

Let $k$ be big enough so that $\left|a_{k}-t\right|<\varsigma / 2$ and $d_{J_{1},[0, t+1]}\left(\bar{S}^{\left(p_{k}\right)}, B\right)<\varsigma / 2$. Thus there exists an increasing homeomorphisms $\varphi_{k}$ of $\left[0, \Delta^{-1}(s)+1\right]$ such that, for all $v \in[0, t+1]$

$$
\begin{aligned}
& \left|v-\varphi_{k}(v)\right|<\frac{\varsigma}{2} \\
& \left|\bar{S}^{\left(p_{k}\right)}(v)-B\left(\varphi_{k}(v)\right)\right|<\frac{\varsigma}{2} .
\end{aligned}
$$

Hence

$$
\left|\bar{S}^{\left(p_{k}\right)}\left(a_{k}\right)-B(t)\right| \leq \frac{\varsigma}{2}+\left|B\left(\varphi_{k}\left(a_{k}\right)\right)-B(t)\right|<\frac{\varsigma}{2}+\frac{\eta}{2}<\eta
$$

since $\left|\varphi_{k}\left(a_{k}\right)-t\right| \leq\left|\varphi_{k}\left(a_{k}\right)-a_{k}\right|+\left|a_{k}-t\right|<\varsigma$.

Assume moreover that $k$ is so big that

$$
\begin{aligned}
& d_{J_{1},[0,|B(t)|+1]}\left(\bar{\omega}^{\left(p_{k}\right)}, Z\right)<\frac{\eta}{2} \\
& d_{J_{1},[0,|B(t)|+1]}\left(\bar{\omega}^{\left(p_{k}\right)}(-(\cdot)), Z(-(\cdot))\right)<\frac{\eta}{2} .
\end{aligned}
$$

Then there exists a strictly increasing homeomorphism $\psi_{k}$ of $[-|B(t)|-1,|B(t)|+1]$, with $\psi_{k}(0)=0$, such that, for all $w \in[-|B(t)|-1,|B(t)|+1]$,

$$
\begin{aligned}
& \left|w-\psi_{k}(w)\right|<\frac{\eta}{2} \\
& \left|\bar{\omega}^{\left(p_{k}\right)}(w)-Z\left(\psi_{k}(w)\right)\right|<\frac{\eta}{2} .
\end{aligned}
$$

Therefore $\left|\bar{\omega}^{\left(p_{k}\right)}\left(\bar{S}^{\left(p_{k}\right)}\left(a_{k}\right)\right)-Z\left(\psi_{k}\left(\bar{S}^{\left(p_{k}\right)}\left(a_{k}\right)\right)\right)\right|<\eta / 2$ and

$$
\begin{aligned}
& \left|\psi_{k}\left(\bar{S}^{\left(p_{k}\right)}\left(a_{k}\right)\right)-B(t)\right| \\
& \quad \leq\left|\psi_{k}\left(\bar{S}^{\left(p_{k}\right)}\left(a_{k}\right)\right)-\bar{S}^{\left(p_{k}\right)}\left(a_{k}\right)\right|+\left|\bar{S}^{\left(p_{k}\right)}\left(a_{k}\right)-B(t)\right| \\
& \quad<2 \eta .
\end{aligned}
$$


whence, by (3.18),

$$
\begin{aligned}
\mid \bar{\omega}^{\left(p_{k}\right)} & \left(\bar{S}^{\left(p_{k}\right)}\left(a_{k}\right)\right)-Z(B(t)) \mid \\
& \leq \frac{\eta}{2}+\left|Z\left(\psi_{k}\left(\bar{S}^{\left(p_{k}\right)}\left(a_{k}\right)\right)\right)-Z(B(t))\right| \\
& <\frac{\eta}{2}+\frac{\varepsilon}{2}<\varepsilon .
\end{aligned}
$$

We conclude that $\lim _{k \rightarrow \infty} \bar{\omega}^{\left(p_{k}\right)}\left(\bar{S}^{\left(p_{k}\right)}\left(a_{k}\right)\right)=Z(B(t))$.

We are now ready to complete the proofs of Theorems 2.1 and 2.2 .

Proof of Theorem [2.1. Let $m \in \mathbb{Z}^{+}$and $s_{1}, \ldots, s_{m} \in[0,+\infty)$ and notice that with probability one, $Z$ is continuous at $B\left(\Delta^{-1}\left(s_{i}\right)\right)$, for every $i \in\{1, \ldots, m\}$.

From the convergence stated in Lemma [3.2, for every $i \in\{1, \ldots, m\}$ we can apply Lemma 3.3 twice: first with $a_{k}=\left(\bar{T}^{\left(p_{k}\right)}\right)^{-1}\left(s_{i}\right)$ and $t=\Delta^{-1}\left(s_{i}\right)$, then with $a_{k}=\left(\bar{T}^{\left(p_{k}\right)}\right)^{-1}\left(s_{i}\right)-q_{k}^{-2 \alpha /(\alpha+1)}$ and again $t=\Delta^{-1}\left(s_{i}\right)$. We then obtain, for all $i=1 \ldots, m$,

$$
\begin{aligned}
\lim _{k \rightarrow \infty} \bar{\omega}^{\left(p_{k}\right)}\left(\bar{S}^{\left(p_{k}\right)}\left(\left(\bar{T}^{\left(p_{k}\right)}\right)^{-1}\left(s_{i}\right)\right)\right) & =\lim _{k \rightarrow \infty} \bar{\omega}^{\left(p_{k}\right)}\left(\bar{S}^{\left(p_{k}\right)}\left(\left(\bar{T}^{\left(p_{k}\right)}\right)^{-1}\left(s_{i}\right)-p_{k}^{-1}\right)\right) \\
& =Z\left(B\left(\Delta^{-1}\left(s_{i}\right)\right)\right) .
\end{aligned}
$$

Hence, from (3.10), it follows that, almost surely,

$$
\lim _{k \rightarrow \infty}\left(\bar{X}^{\left(q_{k}\right)}\left(s_{1}\right), \ldots, \bar{X}^{\left(q_{k}\right)}\left(s_{m}\right)\right)=\left(Z\left(B\left(\Delta^{-1}\left(s_{1}\right)\right)\right), \ldots, Z\left(B\left(\Delta^{-1}\left(s_{m}\right)\right)\right)\right) .
$$

The convergence then holds in distribution, for any choice of $\left(q_{k}\right)$. This concludes the proof of Theorem 2.1.

Proof of Theorem 2.2. Let $m \in \mathbb{Z}^{+}$and $t_{1}, \ldots, t_{m} \in[0,+\infty)$. Fix an arbitrary sequence $q_{k} \rightarrow+\infty$. It suffices to prove the assertion of the theorem for $q=q_{k}$.

For every $i \in\{1, \ldots, m\}$ we get by definition (3.3) that

$$
\frac{T_{\left\lfloor t_{i} q\right\rfloor}}{q^{(\alpha+1) / 2 \alpha}}=\bar{T}^{(q)}\left(t_{i}\right) .
$$

Also, combining definitions (3.1)-(3.2) as in (3.9) gives

$$
\frac{Y_{\left.\left\lfloor t_{i}\right\rfloor\right\rfloor}}{q^{1 / 2 \alpha}}=\bar{\omega}^{(q)}\left(\bar{S}^{(q)}\left(t_{i}\right)\right) .
$$

Now, with probability one $Z$ is continuous at $B\left(t_{1}\right), \ldots, B\left(t_{m}\right)$. We restrict to such realizations. Fix a sequence $q_{k} \rightarrow+\infty$. We use (3.31) with the almost sure version of the Main Lemma 3.1, for $q=q_{k}$, and (3.32) with Lemma 3.3, for $p_{k}=q_{k}$ and $a_{k}=t=t_{i}$. This proves the a.s. convergence of the 1.h.sides of (3.31)-(3.32), along the sequence $\left(q_{k}\right)$, to $\Delta\left(t_{i}\right)$ and $Z\left(B\left(t_{i}\right)\right)$, respectively. Considering the joint convergence for all $i \in\{1, \ldots, m\}$ and passing to distributional convergence proves the desired assertion. 


\section{Proof of the Main Lemma}

The proof of Lemma 3.1 follows a standard argument for the convergence of processes and will be split into two parts: convergence of the finite-dimensional distributions and tightness. Beyond technicalities, the main ingredient of the proof is a representation of the collision time as a suitable random walk in random scenery. Before getting to the proofs proper, it is worth clarifying this idea, introducing certain quantities and giving some estimates that will be used further on.

4.1. Collision time and random scenery. Let us first rewrite $T_{n}$ as

$$
T_{n}:=\sum_{y \in \mathbb{Z}} \mathcal{N}_{n}(y) \zeta_{y},
$$

where $\mathcal{N}_{n}(y)$ denotes the local time on the bonds for the underlying random walk:

$$
\mathcal{N}_{n}(y):=\#\left\{k \in\{1, \ldots, n\}:[y-1, y] \subseteq\left[S_{k-1}, S_{k}\right]\right\} .
$$

In other words, $\mathcal{N}_{n}(y)$ is the number of times, up to the $n^{\text {th }}$ jump, that the walk $X$ travels the $y^{\text {th }}$ gap $\left[\omega_{y-1}, \omega_{y}\right]$. Notice that, given $S, T_{n}$ is a sum of independent random variables. In particular, eq. (4.1) provides an interpretation of the collision time as a random walk in random scenery, and suggests the convergence stated in the Main Lemma. To formalize this connection it is useful to introduce more quantities pertaining to the underlying random walk $S$, such as the standard local time, or local time on the sites:

$$
N_{n}(y):=\#\left\{k \in\{0, \ldots, n-1\}: S_{k}=y\right\}
$$

the range:

$$
R_{n}:=\#\left\{y \in \mathbb{Z}: N_{n}(y)>0\right\}
$$

and the self-intersection:

$$
V_{n}:=\sum_{k, \ell=0}^{n-1} \mathbf{1}_{\left\{S_{k}=S_{\ell}\right\}}=\sum_{y \in \mathbb{Z}} \sum_{k, \ell=0}^{n-1} \mathbf{1}_{\left\{S_{k}=S_{\ell}=y\right\}}=\sum_{y \in \mathbb{Z}}\left(N_{n}(y)\right)^{2} .
$$

We recall (see, e.g., [DE]) that

$$
\mathbb{E}\left[R_{n}\right]=O(\sqrt{n}) .
$$

Moreover, as noticed in [KS], the local limit theorem for $S$ implies that

$$
\mathbb{E}\left[V_{n}\right]=\sum_{k, \ell=0}^{n-1} \mathbb{P}\left(S_{k}=S_{\ell}\right)=n+2 \sum_{0 \leq k<\ell \leq n-1} \mathbb{P}\left(S_{\ell-k}=0\right)=O\left(n^{3 / 2}\right) .
$$

Therefore, by Hölder's inequality, for every $\beta \in(0,2]$ we have

$$
\begin{aligned}
\sum_{y \in \mathbb{Z}} \mathbb{E}\left[\left(N_{n}(y)\right)^{\beta}\right] & \leq\left(\mathbb{E}\left[\sum_{y \in \mathbb{Z}} \mathbf{1}_{\left\{N_{n}(y)>0\right\}}\right]\right)^{1-\beta / 2}\left(\mathbb{E}\left[\sum_{y \in \mathbb{Z}}\left(N_{n}(y)\right)^{2}\right]\right)^{\beta / 2} \\
& =\left(\mathbb{E}\left[R_{n}\right]\right)^{1-\beta / 2}\left(\mathbb{E}\left[V_{n}\right]\right)^{\beta / 2}=O\left(n^{(1+\beta) / 2}\right) .
\end{aligned}
$$


For later purposes we study certain properties of the local time $N_{n}$. To state the first result, we need to introduce $\tau_{n}(x)$, the time of the $n^{\text {th }}$ visit to $x \in \mathbb{Z}$. We define $\tau_{0}(x):=0$ and, for $n \geq 1$,

$$
\tau_{n}(x):=\inf \left\{m>\tau_{n-1}(x): S_{m}=x\right\} .
$$

Then we have the following:

Lemma 4.1. For every $\beta \in(0,1]$, there exists $K_{0}>0$ such that, for every $x, y \in \mathbb{Z}$,

$$
\begin{aligned}
\mathbb{E}\left[\left|N_{n}(x)-N_{n}(y)\right|^{\beta}\right] \leq & K_{0}\left(|x-y|^{\beta / 2} \mathbb{E}\left[\left(N_{n}(x)\right)^{\beta / 2}+\left(N_{n}(y)\right)^{\beta / 2}\right]\right. \\
& \left.+\left(1+(|x-y| \log n)^{\beta}\right)\left(\mathbb{P}\left(\tau_{1}(x) \leq n\right)+\mathbb{P}\left(\tau_{1}(y) \leq n\right)\right)\right) .
\end{aligned}
$$

Proof. As in [KS], for $x, y \in \mathbb{Z}$, we let $M_{j}(x, y)$ denote the number of visits of the random walk $S$ to $y$ between the $j^{\text {th }}$ and the $(j+1)^{\text {th }}$ visit to $x$ :

$$
M_{j}(x, y):=\sum_{m=\tau_{j}(x)+1}^{\tau_{j+1}(x)} \mathbf{1}_{\left\{S_{m}=y\right\}} .
$$

Note that $M_{j}(x, y)$ is independent of the $\sigma$-algebra $\mathcal{F}_{\tau_{j}(x)}$, where $\mathcal{F}_{n}:=\sigma\left(S_{1}, \ldots, S_{n}\right)$. For reasons of symmetry, it is enough to study

$$
\mathbb{E}\left[\left|N_{n}(x)-N_{n}(y)\right|^{\beta} \mathbf{1}_{\left\{\tau_{1}(x) \leq \tau_{1}(y)\right\}}\right] .
$$

Let us observe that

$$
\begin{aligned}
& \left(N_{n}(x)-N_{n}(y)\right) \mathbf{1}_{\left\{\tau_{1}(x) \leq \tau_{1}(y)\right\}} \\
& \quad=\left(\mathbf{1}_{\left\{\tau_{1}(x) \leq n\right\}}+\sum_{j=1}^{N_{n}(x)-1}\left(1-M_{j}(x, y)\right)-\sum_{k=\tau_{N_{n}(x)}(x)}^{n} \mathbf{1}_{\left\{S_{k}=y\right\}}\right) \mathbf{1}_{\left\{\tau_{1}(x) \leq \tau_{1}(y)\right\}} .
\end{aligned}
$$

Using that, for all $a, b \in \mathbb{C}$ and $\beta \in[0,1]$,

$$
|a+b|^{\beta} \leq|a|^{\beta}+|b|^{\beta},
$$

we obtain

$$
\mathbb{E}\left[\left(N_{n}(x)-N_{n}(y)\right)^{\beta} \mathbf{1}_{\left\{\tau_{1}(x) \leq \tau_{1}(y)\right\}}\right] \leq \mathbb{P}\left(\tau_{1}(x) \leq n\right)+\mathcal{A}_{n}(x, y)+\mathcal{B}_{n}(x, y),
$$

where

$$
\begin{aligned}
& \mathcal{A}_{n}(x, y):=\mathbb{E}\left[\left(\sum_{j=1}^{N_{n}(x)-1}\left(1-M_{j}(x, y)\right)\right)^{\beta} \mathbf{1}_{\left\{\tau_{1}(x) \leq \tau_{1}(y)\right\}}\right] \\
& \mathcal{B}_{n}(x, y):=\mathbb{E}\left[\left(\sum_{k=\tau_{N_{n}(x)}(x)}^{n} 1_{\left\{S_{k}=y\right\}}\right)^{\beta} \mathbf{1}_{\left\{\tau_{1}(x) \leq \tau_{1}(y) \leq n\right\}}\right] .
\end{aligned}
$$


By the independence of $\left(M_{j}(x, y), j \geq 1\right)$ and $\tau_{1}(x)$ we get

$$
\begin{aligned}
\mathcal{B}_{n}(x, y) \leq \mathcal{B}_{n}^{\prime}(x, y) & :=\mathbb{E}\left[\max _{1 \leq j \leq n}\left|M_{j}(x, y)\right|^{\beta} \mathbf{1}_{\left\{\tau_{1}(x) \leq n\right\}}\right] \\
& \leq \mathbb{P}\left(\tau_{1}(x) \leq n\right) \mathbb{E}\left[\max _{1 \leq j \leq n}\left|M_{j}(x, y)\right|^{\beta}\right]
\end{aligned}
$$

Moreover, by [KS, proof of Lem. 2], it turns out that the random variables $M_{j}(x, y)$ are mutually independent, with distribution given by the product of a geometric random variable and a Bernoulli random variable, independent and both with parameter $p(|x-y|)$, where $p: \mathbb{N} \longrightarrow[0,1]$ is a function such that $p(r) \sim c r^{-1}$, for some $c>0$, as $r \rightarrow+\infty$. In particular, the variables $M_{j}(x, y)$ are stochastically dominated by independent geometric variables $\widetilde{M}_{j}(x, y)$ with parameter $p(|x-y|)$, that in turn are dominated by independent exponential variables $M_{j}^{\prime}(x, y)$ with parameter $\lambda:=\log (1-p(|x-y|))$. Therefore, by Jensen's inequality and since $\beta \leq 1$,

$$
\begin{aligned}
\mathbb{E}\left[\max _{1 \leq j \leq n}\left|M_{j}(x, y)\right|^{\beta}\right] & \leq 1+\mathbb{E}\left[\max _{1 \leq j \leq n}\left|M_{j}^{\prime}(x, y)\right|^{\beta}\right] \\
& \leq 1+\mathbb{E}\left[\max _{1 \leq j \leq n} M_{j}^{\prime}(x, y)\right]^{\beta} .
\end{aligned}
$$

But

$$
\begin{aligned}
\mathbb{E}\left[\max _{1 \leq j \leq n} M_{j}^{\prime}(x, y)\right] & =\int_{0}^{+\infty} \mathbb{P}\left(\max _{1 \leq j \leq n} M_{j}^{\prime}(x, y) \geq t\right) d t \\
& =\int_{0}^{+\infty}\left(1-\mathbb{P}\left(\max _{1 \leq j \leq n} M_{j}^{\prime}(x, y)<t\right)\right) d t \\
& =\int_{0}^{+\infty}\left(1-\left(1-e^{-\lambda t}\right)^{n}\right) d t \\
& =\int_{0}^{+\infty} e^{-\lambda t} \sum_{k=0}^{n-1}\left(1-e^{-\lambda t}\right)^{k} d t \\
& =\sum_{k=0}^{n-1}\left[\frac{\left(1-e^{-\lambda t}\right)^{k+1}}{\lambda(k+1)}\right]_{0}^{+\infty} \\
& =\sum_{k=1}^{n} \frac{1}{k \lambda} \leq \frac{\log n+1}{\lambda} .
\end{aligned}
$$

Combining (4.17), (4.18) and (4.19) and using the fact that. when $|x-y| \rightarrow+\infty$, $\lambda=\log (1-p(|x-y|) \sim p(|x-y|)) \sim c|x-y|^{-1}$, we obtain the existence of $K_{1}>0$ such that

$$
\mathcal{B}_{n}(x, y) \leq \mathcal{B}_{n}^{\prime}(x, y) \leq K_{1} \mathbb{P}\left(\tau_{1}(x) \leq n\right)(\log n|x-y|)^{\beta}
$$


Concerning the term $\mathcal{A}_{n}(x, y)$ in (4.15), observe that

$$
\sum_{j=1}^{N_{n}(x)-1}\left(1-M_{j}(x, y)\right) \mathbf{1}_{\left\{\tau_{1}(x) \leq \tau_{1}(y)\right\}}=\sum_{j=1}^{n}\left(1-M_{j}(x, y)\right) \mathbf{1}_{\left\{\tau_{j}(x) \leq n\right\}} \mathbf{1}_{\left\{\tau_{1}(x) \leq \tau_{1}(y)\right\}},
$$

and recall that, by $\left[\mathrm{KS}\right.$, Lem. 2], the process $\left(\mathcal{M}_{m}:=\sum_{j=1}^{m} h_{j}, m \geq 1\right)$, with

$$
h_{j}:=\left(1-M_{j}(x, y)\right) \mathbf{1}_{\left\{\tau_{j}(x) \leq n\right\}} \mathbf{1}_{\left\{\tau_{1}(x) \leq \tau_{1}(y)\right\}},
$$

is a martingale with respect to $\left(\mathcal{F}_{\tau_{m+1}(x)}, m \geq 1\right)$, thus implying that $\mathbb{E}\left[h_{j}^{2} \mid \mathcal{F}_{\tau_{j}(x)}\right]=$ $\mathbb{E}\left[M_{j}(x, y)^{2} \mid \mathcal{F}_{\tau_{j}(x)}\right]$. Therefore, using Burkholder's inequality [HH, Thm. 2.11], there exists $C_{\beta}>0$ such that

$$
\begin{aligned}
\mathbb{E}\left[\left|\mathcal{M}_{n}\right|^{\beta}\right] \leq & C_{\beta}\left(\mathbb{E}\left[\left(\sum_{j=1}^{n} \mathbb{E}\left[h_{j}^{2} \mid \mathcal{F}_{\tau_{j}(x)}\right]\right)^{\beta / 2}\right]+\mathbb{E}\left[\max _{j \leq n}\left|h_{j}\right|^{\beta}\right]\right) \\
\leq & C_{\beta}\left(\mathbb{E}\left[\left(\sum_{j=1}^{n} \mathbb{E}\left[\left(M_{j}(x, y)\right)^{2} \mid \mathcal{F}_{\tau_{j}(x)}\right] \mathbf{1}_{\left\{\tau_{j}(x) \leq n\right\}}\right)^{\beta / 2}\right]\right. \\
& \left.+\mathbb{E}\left[\left(1+\max _{1 \leq j \leq n}\left|M_{j}\right|^{\beta}\right) \mathbf{1}_{\left\{\tau_{1}(x) \leq n\right\}}\right]\right) .
\end{aligned}
$$

From [KS, Lem. 2], which ensures the existence of a constant $K_{2}>0$ such that $\mathbb{E}\left[\left(M_{j}(x, y)\right)^{2} \mid \mathcal{F}_{\tau_{j}(x)}\right] \leq K_{2}|x-y|$, we finally get

$$
\begin{aligned}
\mathbb{E}\left[\left|\mathcal{M}_{n}\right|^{\beta}\right] & \leq K_{3}\left(\mathbb{E}\left[\left(\sum_{j=1}^{n}|x-y| \mathbf{1}_{\left\{\tau_{j}(x) \leq n\right\}}\right)^{\beta / 2}\right]+\mathcal{B}_{n}^{\prime}(x, y)+\mathbb{P}\left(\tau_{1}(x) \leq n\right)\right) \\
& \leq K_{3}\left(|x-y|^{\beta / 2} \mathbb{E}\left[\left(N_{n}(x)\right)^{\beta / 2}\right]+\mathcal{B}_{n}^{\prime}(x, y)+\mathbb{P}\left(\tau_{1}(x) \leq n\right)\right),
\end{aligned}
$$

for some $K_{3}>0$. Combined with (4.14), (4.20) and (4.21), this ends the proof of Lemma 4.1.

Lemma 4.2. For any $\beta \leq 1$ such that $\gamma>1+1 / \beta$,

$$
\sum_{y \in \mathbb{Z}} \mathbb{E}\left[\left|\sum_{r \geq 0} \mathbb{P}\left(S_{1}<-r\right)\left(N_{n}(y)-N_{n}(y+r)\right)\right|^{\beta}\right]=O\left(n^{1 / 2+\beta / 4}\right)
$$

as $n \rightarrow \infty$. 
Proof. Using (4.13) we have

$$
\begin{aligned}
\sum_{y \in \mathbb{Z}} \mathbb{E}\left[\left|\sum_{r \geq 0} \mathbb{P}\left(S_{1}<-r\right)\left(N_{n}(y)-N_{n}(y+r)\right)\right|^{\beta}\right] \\
\leq \sum_{y \in \mathbb{Z}} \sum_{r \geq 0}\left(\mathbb{P}\left(S_{1}<-r\right)\right)^{\beta} \mathbb{E}\left[\left|N_{n}(y)-N_{n}(y+r)\right|^{\beta}\right] .
\end{aligned}
$$

By Lemma 4.1, this quantity is dominated by

$$
\begin{aligned}
2 K_{0} & \sum_{y \in \mathbb{Z}} \sum_{r \geq 0}\left(\mathbb{P}\left(S_{1}<-r\right)\right)^{\beta}\left(r^{\beta / 2} \mathbb{E}\left[\left|N_{n}(y)\right|^{\beta / 2}\right]+\left(r^{\beta}(\log n)^{\beta}+1\right) \mathbb{P}\left(\tau_{1}(y) \leq n\right)\right) \\
& \leq 2 K_{0} \sum_{r \geq 0}\left(\mathbb{P}\left(S_{1}<-r\right)\right)^{\beta}\left(r^{\beta}+1\right)\left(\sum_{y \in \mathbb{Z}} \mathbb{E}\left[\left|N_{n}(y)\right|^{\beta / 2}\right]+(\log n)^{\beta} \mathbb{E}\left[R_{n}\right]\right) \\
& =O\left(n^{1 / 2+\beta / 4}\right),
\end{aligned}
$$

where the last estimate follows from (4.6) and (4.8), with $\beta$ replaced by $\beta / 2$, and the following application of the Markov inequality:

$$
\sum_{r \geq 0}\left(\mathbb{P}\left(S_{1}<-r\right)\right)^{\beta}\left(r^{\beta}+1\right) \leq\left(\mathbb{E}\left[\left|S_{1}\right|^{\gamma}\right]\right)^{\beta} \sum_{r \geq 0} r^{-\gamma \beta}\left(r^{\beta}+1\right)
$$

because $\gamma \beta-\beta>1$ by hypothesis.

Later we will need certain inequalities for the local time on the bonds $\mathcal{N}_{n}$ that are analogous to the ones already seen for the local time on the sites $N_{n}$. For this we will exploit the following lemma, which estimates the $\alpha$-norm distance between the two types of local times.

\section{Lemma 4.3.}

$$
\sum_{y \in \mathbb{Z}} \mathbb{E}\left[\left|\mathcal{N}_{n}(y)-\mu_{\xi} N_{n}(y)\right|^{\alpha}\right]=O\left(n^{1 / 2+\alpha / 4}\right)
$$

Proof. We write

$$
\mathcal{N}_{n}(y)=\mathcal{N}_{n}^{-}(y)+\mathcal{N}_{n}^{+}(y)
$$

where

$$
\begin{aligned}
& \mathcal{N}_{n}^{-}(y):=\#\left\{k=0, \ldots, n-1: S_{k} \geq y, S_{k+1} \leq y-1\right\} \\
& \mathcal{N}_{n}^{+}(y):=\#\left\{k=0, \ldots, n-1: S_{k+1} \geq y, S_{k} \leq y-1\right\}
\end{aligned}
$$

denote the number of crossings of $[y-1, y]$, respectively, from right to left and from left to right.

Observe that, for fixed $y$, the process

$$
\left(\mathcal{N}_{n}^{-}(y)-\sum_{r \geq 0} N_{n}(y+r) \mathbb{P}\left(S_{1}<-r\right), n \in \mathbb{Z}^{+}\right)
$$


is a martingale with $k^{\text {th }}$ increment given by

$$
d_{k}^{-}(y):=\sum_{r \geq 0}\left(\mathbf{1}_{\left\{S_{k} \leq y-1\right\}}-\mathbb{P}\left(S_{1}<-r\right)\right) \mathbf{1}_{\left\{S_{k-1}=y+r\right\}} .
$$

By Burkholder's inequality [HH, Thm. 2.11], there exists $C_{\alpha}>0$ such that

$$
\mathbb{E}\left[\left|\mathcal{N}_{n}^{-}(y)-\sum_{r \geq 0} N_{n}(y+r) \mathbb{P}\left(S_{1}<-r\right)\right|^{\alpha}\right] \leq C_{\alpha}\left(A_{y}^{-}+B_{y}^{-}\right),
$$

where

$$
\begin{aligned}
& A_{y}^{-}:=\mathbb{E}\left[\left(\sum_{k=1}^{n} \mathbb{E}\left[\left(d_{k}^{-}(y)\right)^{2} \mid S_{k-1}\right]\right)^{\alpha / 2}\right] ; \\
& B_{y}^{-}:=\mathbb{E}\left[\max _{k \leq n}\left|d_{k}^{-}(y)\right|^{\alpha}\right] .
\end{aligned}
$$

Let us estimate these terms. Using (4.13) with $\beta=\alpha / 2<1$, we get for the first term:

$$
\begin{aligned}
\sum_{y \in \mathbb{Z}} A_{y}^{-} & =\sum_{y \in \mathbb{Z}} \mathbb{E}\left[\left(\sum_{k=0}^{n-1} \sum_{r \geq 0} \mathbb{P}\left(S_{1}<-r\right)\left(1-\mathbb{P}\left(S_{1}<-r\right)\right) \mathbf{1}_{\left\{S_{k}=y+r\right\}}\right)^{\alpha / 2}\right] \\
& \leq \sum_{y \in \mathbb{Z}} \mathbb{E}\left[\left(\sum_{k=0}^{n-1} \sum_{r \geq 0} \mathbb{P}\left(S_{1}<-r\right) 1_{\left\{S_{k}=y+r\right\}}\right)^{\alpha / 2}\right] \\
& \leq \sum_{y \in \mathbb{Z}} \mathbb{E}\left[\left(\sum_{r \geq 0} \mathbb{P}\left(S_{1}<-r\right) N_{n}(y+r)\right)^{\alpha / 2}\right] \\
& \leq \sum_{y \in \mathbb{Z}} \mathbb{E}\left[\sum_{r \geq 0}\left(\mathbb{P}\left(S_{1}<-r\right)\right)^{\alpha / 2}\left(N_{n}(y+r)\right)^{\alpha / 2}\right] \\
& \leq\left(\mathbb{E}\left[\left|S_{1}\right|^{\gamma}\right]\right)^{\alpha / 2} \sum_{r \geq 0} r^{-\alpha \gamma / 2} \sum_{y \in \mathbb{Z}} \mathbb{E}\left[\left(N_{n}(y)\right)^{\alpha / 2}\right] \\
& =O\left(n^{1 / 2+\alpha / 4}\right),
\end{aligned}
$$

where in the last estimates we have used the Markov inequality for the variable $\left|S_{1}\right|^{\gamma}$, with $\gamma>2 / \alpha$, and estimate (4.8) with $\beta=\alpha / 2$.

As for the second term, in view of (4.35) and (4.32), we write

$$
\sum_{y \in \mathbb{Z}} B_{y}^{-} \leq \sum_{y \in \mathbb{Z}} \mathbb{E}\left[D_{y}^{-}+E_{y}^{-}\right]
$$


where

$$
\begin{aligned}
D_{y}^{-} & :=\max _{k \leq n}\left|\sum_{r \geq 0} \mathbf{1}_{\left\{S_{k} \leq y-1, S_{k-1}=y+r\right\}}\right|^{\alpha}=\max _{k \leq n} \mathbf{1}_{\left\{S_{k} \leq y-1<y \leq S_{k-1}\right\}} ; \\
E_{y}^{-} & :=\max _{k \leq n}\left|\sum_{r \geq 0} \mathbb{P}\left(S_{1}<-r\right) \mathbf{1}_{\left\{S_{k-1}=y+r\right\}}\right|^{\alpha} \\
& =\max _{k \leq n}\left|F_{S_{1}}\left(y-S_{k-1}\right)\right|^{\alpha} \mathbf{1}_{\left\{S_{k-1} \geq y\right\}} .
\end{aligned}
$$

Here $F_{S_{1}}$ is the distribution function of $S_{1}=\xi_{1}$. The expectation of the first of the two terms above is easily estimated as follows, upon a relabeling of the index $k$ :

$$
\begin{aligned}
\sum_{y \in \mathbb{Z}} \mathbb{E}\left[D_{y}^{-}\right] & =\sum_{y \in \mathbb{Z}} \mathbb{E}\left[\max _{k \leq n-1} \mathbf{1}_{\left\{S_{k+1}<y \leq S_{k}\right\}}\right] \\
& \leq \mathbb{E}\left[\#\left\{y \in \mathbb{Z}: \exists k \leq n-1 \text { such that } S_{k+1}<y \leq S_{k}\right\}\right] \\
& \leq \mathbb{E}\left[\max _{k \leq n-1} S_{k}-\min _{\ell \leq n-1} S_{\ell}\right]=O(\sqrt{n}),
\end{aligned}
$$

having used Doob's maximal inequality and the fact that $\mathbb{E}\left[S_{n}^{2}\right]=v_{\xi} n$. As concerns the expectation of (4.39),

$$
\begin{aligned}
\sum_{y \in \mathbb{Z}} \mathbb{E}\left[E_{y}^{-}\right] & \leq \sum_{y \in \mathbb{Z}} \mathbb{E}\left[\left(F_{S_{1}}\left(y-\min _{\substack{S_{k-1} \geq y \\
k \leq n}} S_{k-1}\right)\right)^{\alpha} \mathbf{1}_{\left\{\exists k \leq n: S_{k-1} \geq y\right\}}\right] \\
& \leq \mathbb{E}\left[\sum_{y=\min _{k \leq n-1} S_{k}}\left[\sum_{y<\min _{k \leq n-1} S_{k}}\left(F_{S_{1}}\left(y-\min _{\substack{S_{k} \geq y \\
k \leq n-1}} S_{k}\right)\right)^{\alpha}\right]\right. \\
& \leq \mathbb{E}\left[\max _{k \leq n-1} S_{k}-\min _{k \leq n-1} S_{k}+1\right]+\sum_{\xi<0}\left(F_{S_{1}}(\xi)\right)^{\alpha}
\end{aligned}
$$

where again we have redefined the index $k$ in the second inequality. Now, in the last bound above, the first term is $O(\sqrt{n})$, as in (4.40), and the second term is $O(1)$, because $\left(F_{S_{1}}(\xi)\right)^{\alpha}=O\left(|\xi|^{-\alpha \gamma}\right)$ by the Markov inequality and the fact that $\alpha \gamma>1$ by hypothesis; cf. (4.27).

Together with (4.37) and (4.40) we conclude that

$$
\sum_{y \in \mathbb{Z}} B_{y}^{-}=O(\sqrt{n})
$$

whence, in light of (4.33) and (4.36),

$$
\sum_{y \in \mathbb{Z}} \mathbb{E}\left[\left|\mathcal{N}_{n}^{-}(y)-\sum_{r \geq 0} N_{n}(y+r) \mathbb{P}\left(S_{1}<-r\right)\right|^{\alpha}\right]=O\left(n^{1 / 2+\alpha / 4}\right) .
$$


Applying (4.13) and Lemma 4.2, both with $\beta=\alpha$ (since $\gamma>2 / \alpha>1+1 / \alpha$ ), we obtain

$$
\sum_{y \in \mathbb{Z}} \mathbb{E}\left[\left|\mathcal{N}_{n}^{-}(y)-N_{n}(y) \sum_{r \geq 0} \mathbb{P}\left(S_{1}<-r\right)\right|^{\alpha}\right]=O\left(n^{1 / 2+\alpha / 4}\right) .
$$

In the same way one can prove that

$$
\sum_{y \in \mathbb{Z}} \mathbb{E}\left[\left|\mathcal{N}_{n}^{+}(y)-N_{n}(y) \sum_{r \geq 0} \mathbb{P}\left(S_{1}>r\right)\right|^{\alpha}\right]=O\left(n^{1 / 2+\alpha / 4}\right) .
$$

Lemma 4.3 then follows from the two previous estimates and (4.13) with $\beta=\alpha$, because $\mu_{\xi}=\mathbb{E}\left[\left|S_{1}\right|\right]=\sum_{r \geq 0} \mathbb{P}\left(\left|S_{1}\right|>r\right)$.

Observation. Lemma 4.3 is the only part of the paper where the assumption $\gamma>$ $2 / \alpha$ is used.

4.2. Finite-dimensional distributions. To prove the convergence of the finitedimensional joint distribution $\left(\bar{\omega}^{(q)}, \bar{S}^{(q)}, \bar{T}^{(q)}\right)$, as $q \rightarrow \infty$, we use the method of the characteristic function, as in [KS].

Notation. In what follows, the asymptotic equivalence $a(\theta) \sim b(\theta)$, as $\theta \rightarrow \theta_{0}$, means that $\lim _{\theta \rightarrow \theta_{0}} a(\theta) / b(\theta)=1$. In case $b\left(\theta_{j}\right)=0$, for $\theta_{j} \rightarrow \theta_{0}$, the equivalence means that $a\left(\theta_{j}\right)=0$, fo all $j$. A similar notation will be used w.r.t. to other limits, such as $q \rightarrow \infty$.

The assumption on the distribution of the gaps $\zeta_{j}$, see beginning of Section 2 , implies that the following limit exists

$$
c_{0}:=\lim _{z \rightarrow+\infty} z^{\alpha} \mathbb{P}\left(\zeta_{1} \geq z\right)>0 .
$$

Equivalently, denoting by $\phi_{\mathcal{X}}$ the characteristic function of a given random variable $\mathcal{X}$, the following relation holds for $\theta \rightarrow 0$ :

$$
1-\phi_{\zeta_{1}}(\theta) \sim-\log \left(\phi_{Z_{1}}(\theta)\right)=c_{1}|\theta|^{\alpha}\left(1-i \tan \frac{\pi \alpha}{2} \operatorname{sgn}(\theta)\right)
$$

where $Z_{1}$ is the $\alpha$-stable variable whose basin of attraction contains $\zeta_{1}$, cf. Section 2 , and $c_{1}:=\Gamma(1-\alpha) c_{0} \cos (\alpha \pi / 2)[\mathrm{IL}, \S 2.2]$. Hence, there exists a continuous increasing function $\epsilon_{0}: \mathbb{R}_{0}^{+} \longrightarrow \mathbb{R}_{0}^{+}$such that $\epsilon_{0}(0)=0$ and, for all $\theta \in \mathbb{R}$,

$$
\left|\phi_{\zeta_{1}}(\theta)-\phi_{Z_{1}}(\theta)\right| \leq|\theta|^{\alpha} \epsilon_{0}(|\theta|) \text {. }
$$

Proof of the convergence of the finite-dimensional distributions. For a given integer $m \geq 1$, we take $3 m$ real numbers $\kappa_{1}, \ldots, \kappa_{m}, \mu_{1}, \ldots, \mu_{m}, \theta_{1}, \ldots, \theta_{m}$, and $3 m$ real numbers $t_{1}, \ldots, t_{m}, s_{1}, \ldots, s_{m}, v_{1}, \ldots, v_{m}$ satisfying $0<t_{1}<\cdots<t_{m}, 0<v_{1}<$ $\ldots<v_{m}$ and $s_{1}<\cdots<s_{m}$. Let us denote by $\varphi^{(q)}$ the characteristic function of

$$
\left(\bar{\omega}^{(q)}\left(s_{1}\right), \ldots, \bar{\omega}^{(q)}\left(s_{m}\right), \bar{S}^{(q)}\left(v_{1}\right), \ldots, \bar{S}^{(q)}\left(v_{m}\right), \bar{T}^{(q)}\left(t_{1}\right), \ldots, \bar{T}^{(q)}\left(t_{m}\right)\right)
$$


evaluated in $\left(\kappa_{1}, \ldots, \kappa_{m}, \mu_{1}, \ldots, \mu_{m}, \theta_{1}, \ldots, \theta_{m}\right)$. Conditioning with respect to $S$, we have

$$
\varphi^{(q)}=\mathbb{E}\left[\mathbb{E}\left[e^{i \sum_{j=1}^{m}\left(\kappa_{j} \bar{\omega}^{(q)}\left(s_{j}\right)+\theta_{j} \bar{T}^{(q)}\left(t_{j}\right)\right)} \mid S\right] e^{i \sum_{j=1}^{m} \mu_{j} \bar{S}^{(q)}\left(v_{j}\right)}\right] .
$$

Setting $\tilde{\kappa}_{j}:=\operatorname{sgn}\left(s_{j}\right) \kappa_{j}$, the inner expectation can be rewritten as

$$
\begin{aligned}
\mathbb{E}\left[e^{i \sum_{j=1}^{m}\left(\kappa_{j} \bar{\omega}^{(q)}\left(s_{j}\right)+\theta_{j} \bar{T}^{(q)}\left(t_{j}\right)\right)} \mid S\right] \\
=\mathbb{E}\left[\exp \left(i \sum_{y \in \mathbb{Z}} \sum_{j=1}^{m}\left(\theta_{j} q^{-(1+\alpha) / 2 \alpha} \mathcal{N}_{\left\lfloor t_{j} q\right\rfloor}(y)+\tilde{\kappa}_{j} q^{-1 / 2 \alpha} \mathbf{1}_{I_{s_{j} \sqrt{q}}}(y)\right) \zeta_{y}\right) \mid S\right] \\
\quad=\mathbb{E}\left[\prod_{y \in \mathbb{Z}} \phi_{\zeta_{1}}\left(\sum_{j=1}^{m}\left(\theta_{j} q^{-(1+\alpha) / 2 \alpha} \mathcal{N}_{\left\lfloor t_{j} q\right\rfloor}(y)+\tilde{\kappa}_{j} q^{-1 / 2 \alpha} \mathbf{1}_{I_{s_{j} \sqrt{q}}}(y)\right)\right) \mid S\right]
\end{aligned}
$$

with

$$
I_{s}:= \begin{cases}(0, s], & s \geq 0 \\ (s, 0], & s<0\end{cases}
$$

Altogether we get

$$
\varphi^{(q)}=\mathbb{E}\left[\prod_{y \in \mathbb{Z}} \phi_{\zeta_{1}}\left(\sum_{j=1}^{m}\left(\theta_{j} q^{-(1+\alpha) / 2 \alpha} \mathcal{N}_{\left\lfloor t_{j} q\right\rfloor}(y)+\tilde{\kappa}_{j} q^{-1 / 2 \alpha} \mathbf{1}_{I_{s_{j} \sqrt{q}}}(y)\right)\right) e^{i \sum_{j=1}^{m} \mu_{j} \bar{S}^{(q)}\left(v_{j}\right)}\right] .
$$

Convention. In the remainder of this proof, for $a \in \mathbb{R}$, we shall use the notation

$$
|a|_{\mathrm{sgn}}^{\alpha}:=|a|^{\alpha} \operatorname{sgn}(a) \text {. }
$$

Also, we shall introduce pairs of quantities, say $A, A_{\mathrm{sgn}}$, which are defined similarly except that a certain expression $|\cdot|^{\alpha}$ in the first definition is replaced by the corresponding expression $|\cdot|_{\mathrm{sgn}}^{\alpha}$ in the second one. If $A, A_{\mathrm{sgn}}$ and $B, B_{\mathrm{sgn}}$ are two such pairs, we shall use the imprecise notation

$$
A_{\sigma}=B_{\sigma}, \quad \text { for } \sigma \in\{, \operatorname{sgn}\}
$$

to mean that $A=B$ and $A_{\text {sgn }}=B_{\text {sgn }}$.

If we set

$$
V^{(q)}(y):=\sum_{j=1}^{m}\left(\theta_{j} q^{-(1+\alpha) / 2 \alpha} \mathcal{N}_{\left\lfloor t_{j} q\right\rfloor}(y)+\tilde{\kappa}_{j} q^{-1 / 2 \alpha} \mathbf{1}_{I_{s_{j} \sqrt{q}}}(y)\right),
$$

the following is an identity by (4.47):

$$
\begin{gathered}
\prod_{y \in \mathbb{Z}} \phi_{Z_{1}}\left(\sum_{j=1}^{m}\left(\theta_{j} q^{-(1+\alpha) / 2 \alpha} \mathcal{N}_{\left\lfloor t_{j} q\right\rfloor}(y)+\tilde{\kappa}_{j} q^{-1 / 2 \alpha} \mathbf{1}_{I_{s_{j} \sqrt{q}}}(y)\right)\right) \\
=\exp \left(-c_{1} \sum_{y \in \mathbb{Z}}\left(\left|V^{(q)}(y)\right|^{\alpha}-i\left|V^{(q)}(y)\right|_{\operatorname{sgn}}^{\alpha} \tan \frac{\pi \alpha}{2}\right)\right) .
\end{gathered}
$$


Therefore, in view of (4.48), we obtain

$$
\begin{aligned}
\mid \varphi^{(q)} & -\mathbb{E}\left[\exp \left(-c_{1} \sum_{y \in \mathbb{Z}}\left(\left|V^{(q)}(y)\right|^{\alpha}-i\left|V^{(q)}(y)\right|_{\text {sgn }}^{\alpha} \tan \frac{\pi \alpha}{2}\right)+i \sum_{j=1}^{m} \mu_{j} \bar{S}^{(q)}\left(v_{j}\right)\right)\right] \mid \\
\leq & \mathbb{E}\left[\sum_{y \in \mathbb{Z}}\left|V^{(q)}(y)\right|^{\alpha} \epsilon_{0}\left(\left|V^{(q)}(y)\right|\right)\right] \\
\leq & \mathbb{E}\left[\sum_{y \in \mathbb{Z}}\left|V^{(q)}(y)\right|^{\alpha}\right] \epsilon_{0}\left(\sum_{j=1}^{m}\left(\left|\theta_{j}\right| t_{m} q^{(\alpha-1) / 2 \alpha}+\left|\kappa_{j}\right| q^{-1 / 2 \alpha}\right)\right),
\end{aligned}
$$

where in the last inequality we have used that $\mathcal{N}_{\left\lfloor t_{j} q\right\rfloor} \leq t_{m} q$. Now, from the definition of $V^{(q)}$, using (4.13) with $\beta=\alpha<1$, we see that the last term in the above inequality is bounded above by

$$
\mathbb{E}\left[\sum_{j=1}^{m}\left(\left|\theta_{j}\right|^{\alpha} q^{-(\alpha+1) / 2} \sum_{y \in \mathbb{Z}}\left(\mathcal{N}_{\left\lfloor t_{j} q\right\rfloor}(y)\right)^{\alpha}+\left|\tilde{\kappa}_{j}\right|^{\alpha} q^{-1 / 2}\left|s_{j}\right|^{\alpha} q^{\alpha / 2}\right)\right] \epsilon_{q},
$$

where $\epsilon_{q}:=\epsilon_{0}\left(\sum_{j=1}^{m}\left(\left|\theta_{j}\right| t_{j} q^{(\alpha-1) / 2 \alpha}+\left|\kappa_{j}\right| q^{-1 / 2 \alpha}\right)\right)$. Notice that $\epsilon_{q} \rightarrow 0$, as $q \rightarrow \infty$. Now, by Lemma 4.3 and (4.8),

$$
\sum_{y \in \mathbb{Z}} \mathbb{E}\left[\left(\mathcal{N}_{n}(y)\right)^{\alpha}\right]=\sum_{y \in \mathbb{Z}} \mu_{\xi}^{\alpha} \mathbb{E}\left[\left(N_{n}(y)\right)^{\alpha}\right]+O\left(n^{1 / 2+\alpha / 4}\right)=O\left(n^{1 / 2+\alpha / 4}\right)
$$

We conclude that, for $q \rightarrow \infty$,

$$
\varphi^{(q)} \sim \mathbb{E}\left[\exp \left(-c_{1} \sum_{y \in \mathbb{Z}}\left(\left|V^{(q)}(y)\right|^{\alpha}-i\left|V^{(q)}(y)\right|_{\operatorname{sgn}}^{\alpha} \tan \frac{\pi \alpha}{2}\right)+i \sum_{j=1}^{m} \mu_{j} \bar{S}^{(q)}\left(v_{j}\right)\right)\right] .
$$

In order to take the limit of the above r.h.s. we define

$$
\widetilde{V}^{(q)}(y):=\sum_{j=1}^{m}\left(\theta_{j} q^{-(1+\alpha) / 2 \alpha} \mu_{\xi} N_{\left.\left\lfloor t_{j}\right\rfloor\right\rfloor}(y)+\tilde{\kappa}_{j} q^{-1 / 2 \alpha} \mathbf{1}_{I_{s_{j} \sqrt{q}}}(y)\right)
$$

Applying

$$
\left.|| a\right|_{\sigma} ^{\alpha}-|b|_{\sigma}^{\alpha}\left|\leq 2^{1-\alpha}\right| a-\left.b\right|^{\alpha}
$$


which holds true for both $\sigma \in\{, \operatorname{sgn}\}$, since $\alpha \leq 1$, and using (4.56), we obtain

$$
\begin{aligned}
& \left.\left|\sum_{y \in \mathbb{Z}}\right| V^{(q)}(y)\right|_{\sigma} ^{\alpha}-\sum_{y \in \mathbb{Z}}\left|\widetilde{V}^{(q)}(y)\right|_{\sigma}^{\alpha} \mid \\
& \quad \leq 2^{1-\alpha} \sum_{y \in \mathbb{Z}}\left|V^{(q)}(y)-\tilde{V}^{(q)}(y)\right|^{\alpha} \\
& \quad \leq 2^{1-\alpha} \sum_{y \in \mathbb{Z}}\left|\sum_{j=1}^{m} \theta_{j} q^{-(1+\alpha) / 2 \alpha}\left(\mathcal{N}_{\left\lfloor t_{j} q\right\rfloor}(y)-\mu_{\xi} N_{\left\lfloor t_{j} q\right\rfloor}(y)\right)\right|^{\alpha} \\
& \leq 2^{1-\alpha} \sum_{y \in \mathbb{Z}} \sum_{j=1}^{m}\left|\theta_{j}\right|^{\alpha} q^{-(1+\alpha) / 2}\left|\mathcal{N}_{\left\lfloor t_{j} q\right\rfloor}(y)-\mu_{\xi} N_{\left\lfloor t_{j} q\right\rfloor}(y)\right|^{\alpha},
\end{aligned}
$$

where in the last step we have again used (4.13) with $\beta=\alpha$. Therefore, by Lemma 4.3 ,

$$
\mathbb{E}\left[\left.\left|\sum_{y \in \mathbb{Z}}\right| V^{(q)}(y)\right|_{\sigma} ^{\alpha}-\sum_{y \in \mathbb{Z}}\left|\widetilde{V}^{(q)}(y)\right|_{\sigma}^{\alpha} \mid\right]=O\left(q^{-\alpha / 4}\right)
$$

Setting

$$
G_{\sigma}^{(q)}:=\sum_{y \in \mathbb{Z}}\left|\widetilde{V}^{(q)}(y)\right|_{\sigma}^{\alpha}
$$

and combining (4.65) with (4.61), we see, by means of the Lebesgue's Dominated Convergence Theorem, that, for $q \rightarrow \infty$,

$$
\varphi^{(q)} \sim \mathbb{E}\left[\exp \left(-c_{1}\left(G^{(q)}-i G_{\mathrm{sgn}}^{(q)} \tan \frac{\pi \alpha}{2}\right)+i \sum_{j=1}^{m} \mu_{j} \bar{S}^{(q)}\left(v_{j}\right)\right)\right] .
$$

We then define

$$
G_{\sigma}:=\int_{\mathbb{R}}\left|\sum_{j=1}^{m}\left(\theta_{j} \mu_{\xi} L_{t_{j}}(y)+\tilde{\kappa}_{j} \mathbf{1}_{I_{s_{j}}}(y)\right)\right|_{\sigma}^{\alpha} d y .
$$

The following lemma will be proved below.

Lemma 4.4. For fixed values of the parameters $\left\{\kappa_{i}, \mu_{i}, \theta_{i}, t_{i}, s_{i}, v_{i}\right\}_{i=1}^{m}$, as described earlier, the random vector $\left(G^{(q)}, G_{\mathrm{sgn}}^{(q)}, \sum_{j=1}^{m} \mu_{j} \bar{S}^{(q)}\left(v_{j}\right)\right)$ converges in distribution, as $q \rightarrow \infty$, to the vector $\left(G, G_{\mathrm{sgn}}, \sum_{j=1}^{m} \mu_{j} B\left(v_{j}\right)\right)$.

It follows from (4.67), Lemma 4.4 and dominated convergence that

$$
\lim _{q \rightarrow \infty} \varphi^{(q)}=\mathbb{E}\left[\exp \left(-c_{1}\left(G-i G_{\mathrm{sgn}} \tan \frac{\pi \alpha}{2}\right)+i \sum_{j=1}^{m} \mu_{j} B\left(v_{j}\right)\right)\right],
$$

which is the characteristic function of

$$
\left(Z\left(s_{1}\right), \ldots, Z\left(s_{m}\right), B\left(v_{1}\right), \ldots, B\left(v_{m}\right), \Delta\left(t_{1}\right), \ldots, \Delta\left(t_{m}\right)\right)
$$

evaluated in $\left(\kappa_{1}, \ldots, \kappa_{m}, \mu_{1}, \ldots, \mu_{m}, \theta_{1}, \ldots, \theta_{m}\right)$. In fact, 


$$
\begin{aligned}
\sum_{j=1}^{m}\left(\kappa_{j} Z\left(s_{j}\right)+\theta_{j} \Delta\left(t_{j}\right)\right)= & \sum_{j=1}^{m} \int_{0}^{+\infty}\left(\kappa_{j} \mathbf{1}_{I_{j}}(x)+\theta_{j} L_{t_{j}}(x)\right) d Z_{+}(x) \\
& +\int_{0}^{+\infty}\left(\kappa_{j} \mathbf{1}_{I_{j}}(-x)+\theta_{j} L_{t_{j}}(-x)\right) d Z_{-}(x)
\end{aligned}
$$

and so, conditionally to $B$,

$$
\mathbb{E}\left[e^{i \sum_{j=1}^{m}\left(\kappa_{j} Z\left(s_{j}\right)+\theta_{j} \Delta\left(t_{j}\right)\right)} \mid B\right]=\exp \left(-c_{1}\left(G-i G_{s g n} \tan \frac{\pi \alpha}{2}\right)\right) .
$$

Proof of Lemma 4.4. We follow the proof of [KS, Lem. 6]. For all $q, \vartheta \in \mathbb{R}^{+}, M \in \mathbb{Z}^{+}$ and $\sigma \in\{, \operatorname{sgn}\}$, we define

$$
\mathcal{V}_{\sigma}(\vartheta, M, q):=\vartheta^{1-\alpha} \sum_{|k| \leq M}|\mathcal{T}(k, q)|_{\sigma}^{\alpha}
$$

where

$$
\mathcal{T}(k, q):=\sum_{j=1}^{m}\left(\sum_{x=\lceil k \vartheta \sqrt{q}\rceil+1}^{\lceil(k+1) \vartheta \sqrt{q}\rceil}\left(\frac{1}{q} \theta_{j} \mu_{\xi} N_{\left\lfloor t_{j} q\right\rfloor}(x)+\frac{1}{\sqrt{q}} \tilde{\kappa}_{j} \mathbf{1}_{I_{s_{j} \sqrt{q}}}(x)\right)\right),
$$

where $\lceil a\rceil$ is the smallest integer larger than or equal to $a$.

As in [KS], we decompose $G_{\sigma}^{(q)}-\mathcal{V}_{\sigma}(\vartheta, M, q)$ as follows:

$$
G_{\sigma}^{(q)}-\mathcal{V}_{\sigma}(\vartheta, M, q)=U_{\sigma}(\vartheta, M, q)+W_{\sigma}^{1}(\vartheta, M, q)+W_{\sigma}^{2}(\vartheta, M, q),
$$

$$
U_{\sigma}(\vartheta, M, q):=\sum_{x \in A_{\vartheta, M, q}}\left|\sum_{j=1}^{m}\left(q^{-(1+\alpha) / 2 \alpha} \theta_{j} \mu_{\xi} N_{\left\lfloor t_{j} q\right\rfloor}(x)+q^{-1 / 2 \alpha} \tilde{\kappa}_{j} \mathbf{1}_{I_{s_{j} \sqrt{q}}}(x)\right)\right|_{\sigma}^{\alpha},
$$

with $A_{\vartheta, M, q}:=\mathbb{Z} \backslash\{\lceil-M \vartheta \sqrt{q}\rceil+1, \ldots,\lceil(M+1) \vartheta \sqrt{q}\rceil\}$;

$$
W_{\sigma}^{1}(\vartheta, M, q):=\sum_{|k| \leq M} \sum_{x \in E_{k, q}} q^{-(1+\alpha) / 2} W_{\sigma}^{1, k, q}(x),
$$

with $E_{k, q}:=\{\lceil k \vartheta \sqrt{q}\rceil+1, \ldots,\lceil(k+1) \vartheta \sqrt{q}\rceil\}$ and

$$
W_{\sigma}^{1, k, q}(x):=\left|\sum_{j=1}^{m} \theta_{j} \mu_{\xi} N_{\left\lfloor t_{j} q\right\rfloor}(x)+\sqrt{q} \tilde{\kappa}_{j} \mathbf{1}_{I_{s_{j} \sqrt{q}}}(x)\right|_{\sigma}^{\alpha}-q^{\alpha}\left(\# E_{k, q}\right)^{-\alpha}|\mathcal{T}(k, q)|_{\sigma}^{\alpha} ;
$$

and finally

$$
W_{\sigma}^{2}(\vartheta, M, q):=\sum_{|k| \leq M}\left(q^{(\alpha-1) / 2}\left(\# E_{k, q}\right)^{1-\alpha}-\vartheta^{1-\alpha}\right)|\mathcal{T}(k, q)|_{\sigma}^{\alpha} .
$$

Convention. With reference to definition (4.77), we convene that for those values of $\vartheta, k, q$ such that $\lceil k \vartheta \sqrt{q}\rceil=\lceil(k+1) \vartheta \sqrt{q}\rceil, E_{k, q}:=\varnothing$. 
By [KS, Lem. 1, eq. (2.11)] applied with $s=\left\lfloor t_{m} q\right\rfloor$ and $A=M \vartheta / \sqrt{t_{m}}$, there exists a function $\eta: \mathbb{R}^{+} \longrightarrow[0,1]$, vanishing at $+\infty$, such that

$$
\begin{array}{rl}
\sup _{q \geq 1 / t_{m}} & \mathbb{P}\left(U_{\sigma}(\vartheta, M, q) \neq 0\right) \\
\leq & \sup _{q \geq 1 / t_{m}} \mathbb{P}\left(\exists x:|x| \geq M \vartheta \sqrt{q}, N_{\left\lfloor t_{m} q\right\rfloor}(x) \neq 0\right)+\mathbf{1}_{\left\{\max \left\{\left|s_{1}\right|,\left|s_{m}\right|\right\} \geq M \vartheta\right\}} \\
\quad & \eta(M \vartheta) .
\end{array}
$$

Let us prove that there exist $K_{1}, K_{2}>0$ such that, for every $M \in \mathbb{Z}^{+}$,

$$
\sup _{q>1} \mathbb{E}\left[\left|W_{\sigma}^{1}(\vartheta, M, q)\right|\right] \leq K_{1} M \vartheta^{1+\alpha / 2}+K_{2} \vartheta .
$$

Using (4.63) and (4.13) (with $\beta=\alpha$ ), we have

$$
\begin{aligned}
2^{\alpha-1} q^{-(1+\alpha) / 2} \mathbb{E}\left[\left|W_{\sigma}^{1, k, q}(x)\right|\right] & \\
\leq & q^{-(1+\alpha) / 2} \mathbb{E}\left[\left|\sum_{j=1}^{m}\left(\theta_{j} \mu_{\xi} N_{\left\lfloor t_{j} q\right\rfloor}(x)+\sqrt{q} \tilde{\kappa}_{j} \mathbf{1}_{I_{s_{j} \sqrt{q}}}(x)\right)-q \frac{\mathcal{T}(k, q)}{\# E_{k, q}}\right|^{\alpha}\right] \\
\leq & q^{-(1+\alpha) / 2} \mathbb{E}\left[\left|\sum_{j=1}^{m} \theta_{j} \mu_{\xi}\left(N_{\left\lfloor t_{j} q\right\rfloor}(x)-\frac{1}{\# E_{k, q}} \sum_{y \in E_{k, q}} N_{\left\lfloor t_{j} q\right\rfloor}(y)\right)\right|^{\alpha}\right] \\
& +q^{-(1+\alpha) / 2} \mathbb{E}\left[\left|\sum_{j=1}^{m} \sqrt{q} \tilde{\kappa}_{j}\left(\mathbf{1}_{I_{s_{j} \sqrt{q}}}(x)-\frac{1}{\# E_{k, q}} \sum_{y \in E_{k, q}} \mathbf{1}_{I_{s_{j} \sqrt{q}}}(y)\right)\right|^{\alpha}\right]
\end{aligned}
$$

Let us sum the r.h.s. of the last inequality over $|k| \leq M$ and $x \in E_{k, q}$. By $[\mathrm{KS}$, eq. (3.10)], the sum of the first term is bounded above by some constant times $M \vartheta^{1+\alpha / 2}$. As for the second term, we observe that the function $E_{k, q} \ni x \mapsto 1_{I_{s_{j} \sqrt{q}}}(x)$ is constant for all values of $|k| \leq M$ except at most one (corresponding to certain cases where the convex hull of $E_{k, q}$ contains $s_{j} \sqrt{q}$ ). Thus, for all non-exceptional values of $k$ and $x \in E_{k, q}$, the term within parentheses is zero. This and a cancelation of the exponents of $q$ show that the sum over $|k| \leq M$ and $x \in E_{k, q}$ of the last term above is of the order of $\vartheta \sum_{j=1}^{m}\left|\kappa_{j}\right|$. So (4.81) is in force.

Now fix $a, b, c \in \mathbb{R}$ and $\varepsilon>0$. By (4.81) and the inequality $\left|e^{i z}-1\right| \leq|z|$, for $z \in \mathbb{R}$, we see that one can find values of $M, \vartheta$ such that $M \vartheta$ is arbitrarily large but $\vartheta$ is so small that

$$
\sup _{q>1} \mathbb{E}\left[\exp \left(i\left(a W^{1}+b W_{\mathrm{sgn}}^{1}\right)(\vartheta, M, q)\right)-1\right]<\varepsilon .
$$

Also, let us verify that, for all $M, \vartheta$, the variable $W_{\sigma}^{2}(\vartheta, M, q)$ converges in probability to 0 as $q \rightarrow \infty$. For the contribution to $W_{\sigma}^{2}(\vartheta, M, q)$ coming from the first term in $\mathcal{T}(k, q)$, cf. (4.79) and (4.74), this is proved in [KS] (see the argument after eq. (3.7) therein). The contribution from the second term of $\mathcal{T}(k, q)$ is bounded by

$$
\sum_{|k| \leq M}\left(\left|q^{(\alpha-1) / 2}\left(\# E_{k, q}\right)^{1-\alpha}-\vartheta^{1-\alpha}\right| \vartheta^{\alpha} \sum_{j=1}^{m}\left|\kappa_{j}\right|^{\alpha}\right)
$$


but, for all $k,\left(q^{(\alpha-1) / 2}\left(\# E_{k, q}\right)^{1-\alpha}-\vartheta^{1-\alpha}\right) \rightarrow 0$, as $q \rightarrow \infty$. Hence, for all large enough $q$ (depending on $M, \vartheta$ ),

$$
\mathbb{E}\left[\exp \left(i\left(a W^{2}+b W_{\text {sgn }}^{2}\right)(\vartheta, M, q)\right)-1\right]<\varepsilon .
$$

Let us introduce the random function

$$
H_{\sigma}(\vartheta, M):=\vartheta^{1-\alpha} \sum_{|k| \leq M}\left|\int_{k \vartheta}^{(k+1) \vartheta} \sum_{j=1}^{m}\left(\theta_{j} \mu_{\xi} L_{t_{j}}(x)+\tilde{\kappa}_{j} \mathbf{1}_{I_{s_{j}}}(x)\right) d x\right|_{\sigma}^{\alpha} .
$$

It is easy to check that, when $M \rightarrow \infty$ and $\vartheta \rightarrow 0^{+}$in such a way that $M \vartheta \rightarrow+\infty$, $H_{\sigma}(\vartheta, M)$ converges almost surely to $G_{\sigma}$, for both labels $\sigma$.

Therefore, by (4.80), (4.83), (4.85) and the limit just discussed, one can find values of $M, \vartheta$ such that, for all sufficiently large $q$,

$$
\begin{aligned}
& \mathbb{E}\left[\left|\exp \left(i\left(a G^{(q)}+b G_{\mathrm{sgn}}^{(q)}\right)\right)-\exp \left(i\left(a \mathcal{V}+b \mathcal{V}_{\mathrm{sgn}}\right)(\vartheta, M, q)\right)\right|\right]<\varepsilon ; \\
& \mathbb{E}\left[\left|\exp \left(i\left(a H+b H_{\mathrm{sgn}}\right)(\vartheta, M)\right)-\exp \left(i\left(a G+b G_{\mathrm{sgn}}\right)\right)\right|\right]<\varepsilon
\end{aligned}
$$

Moreover, by [KS, eqs. (2.4)-(2.8)], when $q \rightarrow \infty$,

$$
\left(\left(\frac{1}{q} \sum_{x=\lceil k \vartheta \sqrt{q}\rceil}^{\lceil(k+1) \vartheta \sqrt{q}\rceil} N_{\left\lfloor t_{j} q\right\rfloor}, \bar{S}^{(q)}\left(v_{j}\right)\right), j=1, \ldots, m\right)
$$

converges in distribution to

$$
\left(\left(\int_{k \vartheta}^{(k+1) \vartheta} L_{t_{j}(x)} d x, B\left(v_{j}\right)\right), j=1, \ldots, m\right) .
$$

We also have the following convergence of Riemann sums:

$$
\lim _{q \rightarrow \infty} \frac{1}{\sqrt{q}} \sum_{x=\lceil k \vartheta \sqrt{q}\rceil}^{\lceil(k+1) \vartheta \sqrt{q}\rceil} \mathbf{1}_{I_{s_{j} \sqrt{q}}}(x)=\int_{k \vartheta}^{(k+1) \vartheta} \mathbf{1}_{I_{s_{j}}}(x) d x .
$$

Therefore the vector $\left(\mathcal{V}(\vartheta, M, q), \mathcal{V}_{\mathrm{sgn}}(\vartheta, M, q), \sum_{j=1}^{m} \mu_{j} \bar{S}^{(q)}\left(v_{j}\right)\right)$ converges in distribution to $\left(H(\vartheta, M), H_{\mathrm{sgn}}(\vartheta, M), \sum_{j=1}^{m} \mu_{j} B\left(v_{j}\right)\right)$. This means that there exists $q_{0}=q_{0}(M, \vartheta)>0$ such that, for all $q>q_{0}$, (4.87) holds together with the following:

$$
\begin{aligned}
\mathbb{E}\left[\mid \exp \left(i\left(a \mathcal{V}+b \mathcal{V}_{\text {sgn }}\right)(\vartheta, M, q)+i c \sum_{j=1}^{m} \mu_{j} \bar{S}^{(q)}\left(v_{j}\right)\right)\right. \\
\left.\quad-\exp \left(i\left(a H+b H_{\mathrm{sgn}}\right)(\vartheta, M)+i c \sum_{j=1}^{m} \mu_{j} B\left(v_{j}\right)\right) \mid\right]<\varepsilon .
\end{aligned}
$$


Putting the last three inequalities together we obtain that, for all $q>q_{0}$,

$$
\begin{aligned}
\mid \mathbb{E} & {\left[\exp \left(i\left(a G^{(q)}+b G_{\mathrm{sgn}}^{(q)}+c \sum_{j=1}^{m} \mu_{j} \bar{S}^{(q)}\left(v_{j}\right)\right)\right)\right] } \\
& -\mathbb{E}\left[\exp \left(i\left(a G+b G_{\mathrm{sgn}}+c \sum_{j=1}^{m} \mu_{j} B\left(v_{j}\right)\right)\right)\right] \mid<3 \varepsilon .
\end{aligned}
$$

In other words, as $q \rightarrow \infty,\left(G^{(q)}, G_{\mathrm{sgn}}^{(q)}, \sum_{j=1}^{m} \mu_{j} \bar{S}^{(q)}\left(v_{j}\right)\right)$ converges in distribution to $\left(G, G_{\mathrm{sgn}}, \sum_{j=1}^{m} \mu_{j} B\left(v_{j}\right)\right)$.

4.3. Tightness. It is a standard result $\left[\mathrm{S}\right.$ that the sequences $\left(\bar{\omega}^{(q)}(x), x>0\right)_{q \in \mathbb{R}^{+}}$ and $\left(-\bar{\omega}^{(q)}(-x), x>0\right)_{q \in \mathbb{R}^{+}}$, cf. (3.1) , converge in distribution w.r.t. $\left(\mathbb{D}\left(\mathbb{R}_{0}^{+}\right), J_{1}\right)$ to the process $Z_{+}$(or $Z_{-}$) defined in Section 2, In particular they are tight. The same is true for $\left(\bar{S}^{(q)}(t), t \geq 0\right)_{q \in \mathbb{R}^{+}}$, which, due to the functional central limit theorem, converges to $B$.

Hence it remains to prove the tightness of $\left(\bar{T}^{(q)}(t), t \geq 0\right)_{q \in \mathbb{R}^{+}}$on $\left(\mathbb{D}\left(\mathbb{R}_{0}^{+}\right), J_{1}\right)$. Let us fix $T>0$ and prove the tightness of $\left(\bar{T}^{(q)}(t), t \in[0, T]\right)_{q \in \mathbb{R}^{+}}$in $\left(\mathbb{D}[0, T], J_{1}\right)$ following the proof of [KS, Lem. 7].

For a given $\rho>0$, we set $\zeta_{y, q}=\zeta_{y, q, \rho}:=\zeta_{y} \mathbf{1}_{\left\{\zeta_{y} \leq \rho q^{1 / 2 \alpha}\right\}}$ and define the process

$$
\bar{T}_{0}^{(q)}(t)=\bar{T}_{0}^{(q, \rho)}(t):=q^{-(1+\alpha) / 2 \alpha} \sum_{y \in \mathbb{Z}} \mathcal{N}_{\lfloor t q\rfloor}(y) \zeta_{y, q}
$$

that will approximate the process $\bar{T}^{(q)}$. To control the approximation error first notice that, for any $c>0$,

$$
\begin{aligned}
\mathbb{P}(\exists t & \left.\in[0, T]: \bar{T}^{(q)}(t) \neq \bar{T}_{0}^{(q)}(t)\right) \\
& =\mathbb{P}\left(\exists t \in[0, T]: \sum_{y \in \mathbb{Z}} \mathcal{N}_{\lfloor t q\rfloor}(y)\left(\zeta_{y}-\zeta_{y, q}\right) \neq 0\right) \\
& \leq \mathbb{P}\left(\exists y:|y| \leq c \sqrt{q}, \zeta_{y} \neq \zeta_{y, q}\right)+\mathbb{P}\left(\exists t \in[0, T], \exists y:|y|>c \sqrt{q}, \mathcal{N}_{\lfloor t q\rfloor}(y)>0\right) .
\end{aligned}
$$

Now, by virtue of standard results on the maximum of a random walk, we fix $c>0$ such that

$$
\mathbb{P}\left(\exists t \in[0, T], \exists y:|y|>c \sqrt{q}, \mathcal{N}_{\lfloor t q\rfloor}(y)>0\right)=\mathbb{P}\left(\max _{k \leq\lfloor T\rfloor\rfloor}\left|S_{k}\right|>c \sqrt{q}\right)<\frac{\varepsilon}{4}
$$

We then choose $\rho$ such that, using (4.46) as well,

$$
\mathbb{P}\left(\exists y:|y| \leq c \sqrt{q}, \zeta_{y} \neq \zeta_{y, q}\right) \leq 3 c \sqrt{q} \mathbb{P}\left(\zeta_{1} \neq \zeta_{1, q}\right) \leq 3 c c_{0} \rho^{-\alpha}<\frac{\varepsilon}{4} .
$$

Inserting these estimates in (4.95) we obtain

$$
\mathbb{P}\left(\exists t \in[0, T]: \bar{T}^{(q)}(t) \neq \bar{T}_{0}^{(q)}(t)\right)<\frac{\varepsilon}{2},
$$


showing that it is enough to prove the tightness of $\left(\bar{T}_{0}^{(q)}(t), t \in[0, T]\right)_{q \in \mathbb{R}^{+}}$in $\left(\mathbb{D}[0, T], J_{1}\right)$.

We observe that, due to $[\mathrm{B}$, Thm. 13.5, eq. (13.14)], it is enough to prove the existence of $K_{1}>0$ such that, for every $s, t$ with $0<s<t<T$,

$$
\mathbb{E}\left[\left|\bar{T}_{0}^{(q)}(t)-\bar{T}_{0}^{(q)}(s)\right|\right] \leq K_{1}\left(q^{-1}(\lfloor t q\rfloor-\lfloor s q\rfloor)\right)^{3 / 4}
$$

Indeed, together with Hölder's inequality, this would imply that for every $0<s<$ $r<t<T$,

$$
\mathbb{E}\left[\left|\bar{T}_{0}^{(q)}(r)-\bar{T}_{0}^{(q)}(s)\right|^{1 / 2}\left|\bar{T}_{0}^{(q)}(t)-\bar{T}_{0}^{(q)}(r)\right|^{1 / 2}\right] \leq K_{1}(2(t-s))^{3 / 4}
$$

The above uses that, when $t-s \geq 1 / q,\lfloor t q\rfloor-\lfloor s q\rfloor \leq 2 q(t-s)$ and, when $t-s<1 / q$, either $\lfloor r q\rfloor=\lfloor s q\rfloor$ or $\lfloor r q\rfloor=\lfloor t q\rfloor$ making the l.h.s. of (4.100) null.

To verify (4.99), we decompose $\bar{T}_{0}^{(q)}$ in $\bar{T}_{1}^{(q)}+\bar{T}_{2}^{(q)}$, where

$$
\begin{aligned}
& \bar{T}_{1}^{(q)}(t):=q^{-(1+\alpha) / 2 \alpha} \sum_{y \in \mathbb{Z}} \mathcal{N}_{\lfloor t q\rfloor}(y) \mathbb{E}\left[\zeta_{1, q}\right] \\
& \bar{T}_{2}^{(q)}(t):=q^{-(1+\alpha) / 2 \alpha} \sum_{y \in \mathbb{Z}} \mathcal{N}_{\lfloor t q\rfloor}(y) \bar{\zeta}_{y, q},
\end{aligned}
$$

with $\bar{\zeta}_{y, q}:=\zeta_{y, q}-\mathbb{E}\left[\zeta_{1, q}\right]$. Using (4.46), we see that, as $q \rightarrow \infty$,

$$
\begin{aligned}
\mathbb{E}\left[\zeta_{1, q}\right] & =\int_{0}^{+\infty} \mathbb{P}\left(\zeta_{1, q}>u\right) d u=\int_{0}^{\rho q^{1 / 2 \alpha}}\left(\mathbb{P}\left(\zeta_{1}>u\right)-\mathbb{P}\left(\zeta_{1}>\rho q^{1 / 2 \alpha}\right)\right) d u \\
& \sim c_{0} \frac{\alpha}{1-\alpha} \rho^{1-\alpha} q^{(1-\alpha) / 2 \alpha}=: K_{2} q^{(1-\alpha) / 2 \alpha}
\end{aligned}
$$

for a certain positive constant $K_{2}$. Analogously there exists $K_{3}>0$ such that

$$
\mathbb{E}\left[\zeta_{1, q}^{2}\right]=\int_{0}^{+\infty} \mathbb{P}\left(\zeta_{1, q}^{2}>u\right) d u \leq \int_{0}^{\rho^{2} q^{1 / \alpha}} \mathbb{P}\left(\zeta_{1}>\sqrt{u}\right) d u \sim K_{3} q^{(2-\alpha) / 2 \alpha}
$$

Now, on one hand, since $S$ and $\zeta$ are independent, we obtain

$$
\begin{aligned}
\mathbb{E}\left[\left|\bar{T}_{1}^{(q)}(t)-\bar{T}_{1}^{(q)}(s)\right|\right] & \leq q^{-(1+\alpha) / 2 \alpha} \sum_{y \in \mathbb{Z}} \mathbb{E}\left[\left|\mathcal{N}_{\lfloor t q\rfloor}(y)-\mathcal{N}_{\lfloor s q\rfloor}(y)\right|\right] \mathbb{E}\left[\zeta_{1, q}\right] \\
& \leq q^{-(1+\alpha) / 2 \alpha} \sum_{j=\lfloor s q\rfloor+1}^{\lfloor t q\rfloor} \mathbb{E}\left[\left|S_{j}-S_{j-1}\right|\right] \mathbb{E}\left[\zeta_{1, q}\right] \\
& \leq 2 K_{2} q^{-1}(\lfloor t q\rfloor-\lfloor s q\rfloor) \mathbb{E}\left[\left|S_{1}\right|\right]
\end{aligned}
$$


for every $q$ large enough, by (4.103). On the other hand, using in addition that the variables $\left\{\bar{\zeta}_{y, q}\right\}_{y \in \mathbb{Z}}$ are mutually orthogonal, we write

$$
\begin{aligned}
\mathbb{E}\left[\left(\bar{T}_{2}^{(q)}(t)-\bar{T}_{2}^{(q)}(s)\right)^{2}\right] & \leq q^{-(1+\alpha) / \alpha} \sum_{y \in \mathbb{Z}} \mathbb{E}\left[\left(\mathcal{N}_{\lfloor t q\rfloor}(y)-\mathcal{N}_{\lfloor s q\rfloor}(y)\right)^{2}\right] \mathbb{E}\left[\left(\bar{\zeta}_{1, q}\right)^{2}\right] \\
& \leq q^{-(1+\alpha) / \alpha} \sum_{y \in \mathbb{Z}} \mathbb{E}\left[\left(\mathcal{N}_{\lfloor t q\rfloor-\lfloor s q\rfloor}(y)\right)^{2}\right] \mathbb{E}\left[\left(\zeta_{1, q}\right)^{2}\right]
\end{aligned}
$$

At this point observe that (4.33)-(4.36) and (4.42) are still valid if $\alpha=2$. Hence, using the inequality $2 a b \leq a^{2}+b^{2}$ and (4.8) with $\beta=2$, we have, for $n \rightarrow \infty$,

$$
\begin{aligned}
\sum_{y \in \mathbb{Z}} \mathbb{E} & {\left[\left(\mathcal{N}_{n}(y)\right)^{2}\right] } \\
\leq & 2 \sum_{y \in \mathbb{Z}} \mathbb{E}\left[\left(\sum_{r \in \mathbb{Z}} \mathbb{P}\left(\left|S_{1}\right|>r\right) N_{n}(y+r)\right)^{2}\right]+O(n) \\
\leq & \sum_{y \in \mathbb{Z}} \sum_{r, s \in \mathbb{Z}} \mathbb{P}\left(\left|S_{1}\right|>r\right) \mathbb{P}\left(\left|S_{1}\right|>s\right) \mathbb{E}\left[\left(N_{n}(y+r)\right)^{2}+\left(N_{n}(y+s)\right)^{2}\right]+O(n) \\
\leq & 2 \mu_{\xi}^{2} \sum_{y \in \mathbb{Z}} \mathbb{E}\left[\left(N_{n}(y)\right)^{2}\right]+O(n) \\
& =O\left(n^{3 / 2}\right) .
\end{aligned}
$$

Applying the above and (4.104) in (4.106) yields

$$
\mathbb{E}\left[\left(\bar{T}_{2}^{(q)}(t)-\bar{T}_{2}^{(q)}(s)\right)^{2}\right] \leq K_{4} q^{-3 / 2}(\lfloor t q\rfloor-\lfloor s q\rfloor)^{3 / 2},
$$

for some $K_{4}>0$. Therefore, by Jensen's inequality,

$$
\mathbb{E}\left[\left|\bar{T}_{2}^{(q)}(t)-\bar{T}_{2}^{(q)}(s)\right|\right] \leq \sqrt{K_{4}} q^{-3 / 4}(\lfloor t q\rfloor-\lfloor s q\rfloor)^{3 / 4},
$$

which, combined with (4.105), gives (4.99), concluding the proof of the tightness of $\left(\bar{T}^{(q)}\right)_{q \in \mathbb{R}^{+}}$and therefore of Lemma 3.1.

\section{REFERENCES}

[ACOR] R. Artuso, G. Cristadoro, M. Onofri, M. Radice, Non-homogeneous persistent random walks and Lévy-Lorentz gas, J. Stat. Mech. Theory Exp. 2018, no. 8, 083209, 13 pp.

[B] P. Billingsley, Convergence of probability measure, Second edition. John Wiley \& Sons, Inc., New York, 1999.

[BFK] E. Barkai, V. Fleurov, J. Klafter, One-dimensional stochastic Lévy-Lorentz gas, Phys. Rev. E 61 (2000), no. 2, 1164-1169.

[BCll] A. Bianchi, G. Cristadoro, M. Lenci, M. Ligabò, Random walks in a onedimensional Lévy random environment, J. Stat. Phys. 163 (2016), no. 1, 22-40.

[Bo1] A. N. Borodin, A limit theorem for sums of independent random variables defined on a recurrent random walk (in Russian), Dokl. Akad. Nauk SSSR 246 (1979), no. 4, 786-787. 
[Bo2] A. N. Borodin, Limit theorems for sums of independent random variables defined on a transient random walk (in Russian), Investigations in the theory of probability distributions, IV. Zap. Nauchn. Sem. Leningrad. Otdel. Mat. Inst. Steklov. (LOMI) 85 (1979), 17-29, 237, 244.

[BCV] R. Burioni, L. Caniparoli, A. Vezzani, Lévy walks and scaling in quenched disordered media, Phys. Rev. E 81 (2010), 060101(R), 4 pp.

[CGLS] G. Cristadoro, T. Gilbert, M. Lenci, D. P. Sanders, Transport properties of Lévy walks: an analysis in terms of multistate processes, Europhys. Lett. 108 (2014), no. 5, 50002, 6 pp.

[D] R. M. Dudley, Distances of probability measures and random variables, Ann. Math. Statist. 39 (1968), no. 5, 1563-1572.

[DE] A. Dvoretzky, P. Erdös, Some problems on random walk in space, Proceedings of the Second Berkeley Symposium on Mathematical Statistics and Probability, 1950. pp. 353-367. University of California Press, Berkeley and Los Angeles, 1951.

$[\mathrm{HH}] \quad$ P. Hall, C. C. Heyde, Martingale limit theory and its application, Probability and Mathematical Statistics. Academic Press, Inc., New York-London, 1980.

[IL] I. A. Ibragimov, YU. V. Linnik, Independent and stationary sequences of random variables, With a supplementary chapter by I. A. Ibragimov and V. V. Petrov. WoltersNoordhoff Publishing, Groningen, 1971.

[KS] H. Kesten, F. SpItzer, A limit theorem related to a new class of self-similar processes, Z. Wahrsch. Verw. Gebiete 50 (1979), no. 1, 5-25.

[KRS] R. Klages, G. Radons, I. M. Sokolov (eds.), Anomalous Transport: Foundations and Applications, Wiley, Weinheim, 2008.

[Le] P. Levitz, From Knudsen diffusion to Levy walks, Europhys. Lett. 39 (1997), no. 6, 593-598.

[MS] M. Magdziarz, W. Szczotka, Diffusion limit of Lévy-Lorentz gas is Brownian motion, Commun. Nonlinear Sci. Numer. Simul. 60 (2018), 100-106.

[SZU] M. Shlesinger, G. Zaslavsky, U. Frisch (EDs.), Lévy Flights and Related Topics in Physics, Lecture Notes in Physics 450. Springer-Verlag, Berlin, 1995.

[S] A. V. Skorohod, Limit theorems for stochastic processes with independent increments (in Russian), Teor. Veroyatnost. i Primenen. 2 (1957), 145-177.

[VBB] A. Vezzani, E. Barkai, R. Burioni, The Single Big Jump Principle in physical modelling, preprint (2018), arXiv:1804.02932v2.

[ZDK] V. Zaburdaev, S. Denisov, J. Klafter, Lévy walks, Rev. Mod. Phys. 87 (2015), 483-530.

Dipartimento di Matematica, Università di Padova, Via Trieste 63, 35121 Padova, ITALY.

E-mail address: bianchi@math.unipd.it

Dipartimento di Matematica, Università di Bologna, Piazza di Porta San Donato 5, 40126 Bologna, Italy, and Istituto Nazionale di Fisica Nucleare, Sezione di Bologna, Via Irnerio 46, 40126 Bologna, Italy.

E-mail address: marco.lenci@unibo.it

Institut Universitaire de France and Université de Brest, UMR CNRS 6205, LABoratoire De Mathématique de Bretagne Atlantique, 6 Avenue Le Gorgeu, 29238 Brest CEDEX, France.

E-mail address: francoise.pene@univ-brest.fr 